\title{
Thin film modeling of crystal dissolution and growth in confinement
}

\author{
Luca Gagliardi ${ }^{*}$ and Olivier Pierre-Louis ${ }^{\dagger}$ \\ CNRS, ILM Institut Lumière Matière, Université Claude Bernard Lyon 1 Campus LyonTech-La Doua Batiment Brillouin, \\ 10 rue Ada Byron, F-69622 Villeurbanne, France
}

(Received 12 September 2017; published 26 January 2018)

\begin{abstract}
We present a continuum model describing dissolution and growth of a crystal contact confined against a substrate. Diffusion and hydrodynamics in the liquid film separating the crystal and the substrate are modeled within the lubrication approximation. The model also accounts for the disjoining pressure and surface tension. Within this framework, we obtain evolution equations which govern the nonequilibrium dynamics of the crystal interface. Based on this model, we explore the problem of dissolution under an external load, known as pressure solution. We find that in steady state, diverging (power-law) crystal-surface repulsions lead to flat contacts with a monotonic increase of the dissolution rate as a function of the load. Forces induced by viscous dissipation then surpass those due to disjoining pressure at large enough loads. In contrast, finite repulsions (exponential) lead to sharp pointy contacts with a dissolution rate independent of the load and the liquid viscosity. Ultimately, in steady state, the crystal never touches the substrate when pressed against it. This result is independent from the nature of the crystal-surface interaction due to the combined effects of viscosity and surface tension.
\end{abstract}

DOI: 10.1103/PhysRevE.97.012802

\section{INTRODUCTION}

Pressure solution is the stress-induced dissolution of solids in the presence of an applied load. This phenomenon, for example, contributes to the evolution of the structure of sedimentary rocks, which are initially constituted of porous or loose assemblies of grains. When these rocks are under pressure, grains dissolve in areas of relatively high stresses located in contact regions between grains, and reprecipitate elsewhere, leading to a slow compaction of the global structure. Hence, pressure solution is recognized as one of the main mechanism of rock diagenesis and metamorphism. Pressure solution, and the opposite problem of crystallization force (the mechanical stress generated by crystal growth), have attracted attention since the beginning of the 20th century [1-8].

The material dissolved during pressure solution is usually evacuated away from the contact region toward a macroscopic reservoir through the thin liquid film between the crystal surface and a confining substrate (pore surface or other crystals) [9-11]. While the thermodynamic equilibrium description provides fundamental understanding of the forces at play in this problem [11], the nonequilibrium kinetics of the relevant transport processes induce additional complexity via the combined effects of attachment-detachment kinetics at crystal-solution interface, diffusion of solute crystal ions or molecules, and convection.

Following the seminal phenomenological approach by Weyl [12], some theoretical efforts have been devoted to the description of crystal growth and dissolution in grain-grain or grainpore contacts [13-18]. However, one systematic limitation of these studies is the lack of description of microscopic interactions between the solid surface and the substrate, which are

\footnotetext{
*luca.gagliardi@univ-lyon1.fr

†olivier.pierre-louis@univ-lyon1.fr
}

usually described via the disjoining pressure in thin films [19]. These interactions combine with the spontaneous remodeling of the surface via dissolution and growth to determine the contact morphology and dynamics. In past decades, surface force apparatus (SFA) has allowed one to probe interactions between surfaces at the nanoscale. These experiments have provided quantitative tests for standard theories such as the DLVO (Derjaguin, Landau, Verwey and Overbeek) theory $[19,20]$, combining an exponential electrostatic repulsion with a power-law van der Waals attraction. However, if on the one hand double layer repulsion is considered as the prototypical interaction; on the other hand it was found to be accurate at all separations only for smooth crystalline surfaces in dilute electrolyte solutions [21].

For other surfaces and solutions, significant deviation especially at short range (few nanometers) was measured [21-25]. These non-DLVO contributions to the interaction have been found to depend on the specific nature of the surfaces, the solvent, the ions in the solvent, and the ions adsorbed on the surfaces. Using SFA or atomic force microscopy (AFM) on systems relevant to pressure solution such as silica compounds (mica, silica colloids) and soluble salts, different authors have revealed the existence of additional repulsive interactions at short distances (a few nanometers) referred to as hydration forces $[21,26,27]$. These interactions, the exact mathematical form of which is still matter of debate, are often recognized to be exponentially decaying. Beyond hydration forces, other specific interactions include [19] oscillations at the molecular scale due to liquid ordering, solute-induced effects, depletion effects, etc. Owing to this wide variety of behaviors, we aim at developing an approach which is able to relate the form of the interaction potential and the dynamics during pressure solution or growth. In this paper, we focus on generic repulsive interaction potential, such as exponential or power law. Our first goal is to question the role of the form of the interaction potential on the dynamics of pressure solution. 
A second goal is to identify the consequences of hydrodynamic convection in the thin liquid film. Indeed, convection has long been recognized to be important for solids growing with unconstrained interfaces, both in dendritic growth arising from solidification [28], and in growth from a solution [29]. However, its consequences have not been discussed in confined geometries. In the absence of dissolution or growth, the hydrodynamics of squeezed films have been extensively studied in the literature. This is known to lead to an evolution of the thickness of the film exhibiting a nontrivial dependence on the solid geometry and dimensionality [30]. In pressure solution, the geometry of the dissolving surface evolves in time and emerges from a coupling between different forces and mass transport processes at play in the system. However, a complete description of growth with hydrodynamics (see, e.g., Refs. [31-33]) requires considerable numerical effort since it involves the concomitant solutions of the three-dimensional Navier-Stokes equation and of the evolution of the morphology of the crystal-liquid interface.

Here we propose to tackle this problem, accounting consistently for thermodynamics, interaction effects (i.e., disjoining pressure), and nonequilibrium transport processes including diffusion and convection within a thin film approach which exploits the natural geometric slenderness of the contact region via the lubrication approximation [34]. This method leads to a reduction of dimensionality, thereby facilitating numerical and analytical investigations.

The first part this paper in Sec. II presents a threedimensional continuum model which takes into account dissolution or growth, disjoining pressure effects, diffusion, and hydrodynamics. The key assumption that the film is thin in the contact region and is then formalized with the help of a multiscale expansion defining the lubrication limit [34]. This limit, widely employed in engineering (trust bearing) [30], physics (nanoscale dewetting) [35,36], and biophysical models (membranes) [37,38], results in nonlinear and nonlocal thin film evolution equations for the profile of the crystal surface. The end of Sec. II presents equations for pressure solution in single contacts with some simplifying assumptions such as equal densities between the liquid and the solid, imposed symmetry (left-right symmetric ridge or axisymmetric contact), and dilute limit.

Section III is devoted to the discussion of relevant dimensionless numbers, and numerical methods.

In Sec. IV, we focus on the analysis of pressure solution for a single contact. We investigate steady states with a time-independent surface profile and a fixed contact area. We consider two different classes of repulsive interactions between the crystal surface and the substrate: divergent at contact and finite at contact.

The dissolution rate is found to increase indefinitely with increasing load in the case of diverging repulsions. Viscosity effects then become relevant for sufficiently large loads. However, in the case of finite repulsions, the dissolution rate is independent both on the viscosity and on the load at large loads.

Moreover, as expected intuitively, the shape of the solid is flattened in the contact region for diverging repulsions. However, we find sharp and pointy contact shapes for finite repulsions. In the limit of large loads, surface tension is found

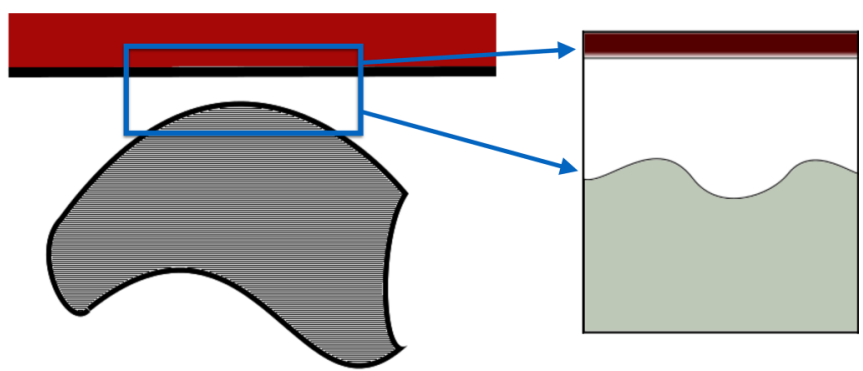

FIG. 1. Sketch of an arbitrarily shaped crystal in the vicinity of a flat substrate. The panel on the right side presents an enlargement of the contact region.

to be irrelevant for diverging repulsions, while it is crucial in the case of finite repulsions to regularize the pointy shapes at small scales.

We have also investigated the effect of dimensionality via the comparison of one-dimensional ridge contacts and twodimensional axisymmetric contacts. Dimensionality does not induce any qualitative change in the behavior of pressure solution for diverging repulsions. However, for finite repulsions and when surface tension is neglected, the minimum distance between the dissolving solid and the substrate decreases exponentially with the load in the ridge geometry, while it reaches zero for a finite force in the axisymmetric case. Surface tension then comes into play at sufficiently large loads and forbids real contact in the axisymmetric geometry.

Finally, the results are summarized and discussed in Sec.V.

\section{MODEL EQUATIONS}

\section{A. Dissolution and growth in a liquid}

The system under study is represented in Figs. 1 and 2. For the sake of clarity, we designate the growing or dissolving solid by the name "crystal." However, our model equally applies to

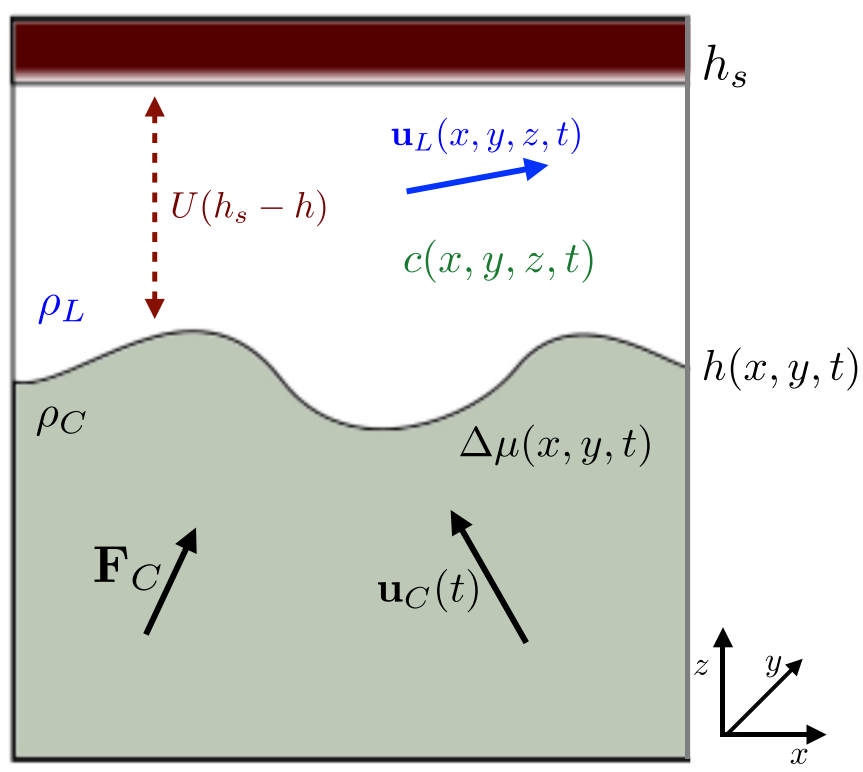

FIG. 2. Sketch of the contact region with some variables and fields of the model. See text for notations. 
amorphous phases or any other solid phases that can grow and dissolve. We consider a crystal in a liquid medium, growing or dissolving in the vicinity of a substrate, and subjected to an external force or load $\mathbf{F}_{C}(t)$. The crystal is assumed to be rigid; that is, we neglect the contribution of elastic deformations on the interface shape and chemical potential. For the sake of simplicity, we also discard crystal rotations and consider only translations. The substrate at $z=h_{s}(x, y)$ is immobile, i.e., $\partial_{t} h_{s}=0$, and impermeable. The liquid crystal interface (LC) at $z=h(x, y, t)$ evolves with time.

We assume an incompressible fluid with constant density $\rho_{L}$

$$
\nabla \cdot \mathbf{u}_{L}=0 .
$$

Neglecting inertial effects (which are known to be negligible in the lubrication limit considered below [34,39]), the liquid obeys the Stokes equation:

$$
\eta \nabla^{2} \mathbf{u}_{L}=-\nabla p
$$

where $\eta$ is the viscosity and $p(x, y, z, t)$ is the pressure. Global mass conservation at the LC interface (neglecting possible mass excess at the interface) reads [29]

$$
\rho_{L}\left(\mathbf{u}_{L} \cdot \hat{\mathbf{n}}-v_{n}\right)=\rho_{C}\left(\mathbf{u}_{C} \cdot \hat{\mathbf{n}}-v_{n}\right),
$$

where $\rho_{C}$ is the constant crystal density, $\mathbf{u}_{C}$ is the translational velocity of the rigid crystal, $\hat{\mathbf{n}}$ is the normal vector of the LC interface, and $v_{n}$ is the normal velocity of the interface. Note that whenever a three-dimensional field such as $\mathbf{u}_{L}$ appears in an equation evaluated at an interface, we consider implicitly the value of this field at this interface. Finally, we assume no slip and no penetrability at the liquid-substrate (LS) interface

$$
\mathbf{u}_{L}=\mathbf{0}
$$

and a no-slip condition at the LC interface

$$
\mathbf{u}_{L \|}=\mathbf{u}_{C \|},
$$

where the index $\|$ indicates the projection of a vector on the plane tangent to the LC interface.

Local mass conservation of the solute (crystal ions or molecules in the fluid) reads in the liquid bulk

$$
\partial_{t} c+\mathbf{u}_{L} \cdot \nabla c=-\nabla \cdot \mathbf{j},
$$

where $\mathbf{j}$ is the diffusion flux. We assume that diffusion is governed by Fick's law

$$
\mathbf{j}=-D(c) \nabla c .
$$

At the LC interface, solute mass conservation imposes

$$
\Omega^{-1}\left(v_{n}-\hat{\mathbf{n}} \cdot \mathbf{u}_{C}\right)=c\left(v_{n}-\hat{\mathbf{n}} \cdot \mathbf{u}_{L}\right)-\hat{\mathbf{n}} \cdot \mathbf{j},
$$

where $\Omega$ is the molecular volume in the crystal.

Assuming that the substrate is impermeable at the LS interface, we have

$$
\mathbf{j} \cdot \hat{\mathbf{n}}_{s}=0,
$$

with $\hat{\mathbf{n}}_{s}$ being the LS interface normal.

The crystallization-dissolution rate $v_{n}-\mathbf{n} \cdot \mathbf{u}_{C}$ is assumed to depend linearly on the departure from equilibrium

$$
v_{n}-\mathbf{n} \cdot \mathbf{u}_{C}=\Omega v\left(c-c_{e q}\right),
$$

where $v$ is a kinetic coefficient and $c_{e q}(x, y, t)$ the local equilibrium concentration. In the ideal limit, where the activity coefficient is equal to 1 , we have

$$
c_{e q}=c_{0} e^{\Delta \mu / k_{B} T},
$$

where $\Delta \mu$ is the local chemical potential of the crystal at the interface and $c_{0}$ is the equilibrium concentration for an interface in an infinitely large crystal far from the substrate (solubility). The chemical potential at the LC interface reads

$$
\frac{\Delta \mu(x, y, t)}{\Omega}=\tilde{\gamma}: \kappa+W^{\prime}(x, y, h)+\left(\frac{\rho_{C}}{\rho_{L}}-1\right) \sigma_{n n},
$$

where $\tilde{\gamma}(x, y)$ is the stiffness tensor [40], $\kappa$ is the curvature tensor, and $W^{\prime}=\partial_{h} W(x, y, h)$ is the disjoining pressure [41]. The potential $W(x, y, h)$ is taken to depend on $x$ and $y$ to account for the possible spatial heterogeneities of the substrate height $h_{s}$ and of the substrate material properties. The liquid stress tensor components are defined as $\sigma_{i j}=\sigma_{i j}^{\prime}-\delta_{i j} p$ with $\sigma_{i j}^{\prime}=\eta\left(\partial_{j} u_{L i}+\partial_{i} u_{L j}\right)$, and the index $n$ indicates the normal direction. The last term of Eq. (12) accounts for the energy cost associated to the volume change during the phase transformation.

Finally, since the crystal is a rigid body, and since we neglect inertia, we write a global force balance on the crystal as

$$
\mathbf{F}_{C}=\oiint_{L C} d S\left[-\hat{\mathbf{n}} \cdot \sigma+\hat{\mathbf{n}}\left(\tilde{\gamma}: \kappa+W^{\prime}\right)\right],
$$

where the surface integral is performed along all the LC interface ( since we discard crystal rotations, we do not consider the equilibrium of torques).

The system of equations reported above describes the dissolution or growth dynamics of a rigid crystal interacting with a frozen and impermeable substrate. In the following, we specialize the discussion to the contact region.

\section{B. Contact region}

In this section, we rewrite mass conservation and force balance in a form which makes use of the geometry of the contact region. We assume that the LS and LC interfaces exhibit no overhang.

For any field $g(x, y, z)$ defined everywhere in the liquid, we consider the following integrated quantity along $z$ :

$$
\langle g\rangle(x, y)=\int_{h(x, y)}^{h_{s}(x, y)} d z g(x, y, z) .
$$

Using the incompressibility condition, Eq. (1), the immobility of the substrate, Eq. (4), and global mass conservation at the LC interface, Eq. (3), we obtain a two-dimensional equation for liquid mass conservation:

$$
\frac{\rho_{C}}{\rho_{L}} v_{C z}=-\nabla_{x y} \cdot\left\langle\mathbf{u}_{L x y}\right\rangle-\partial_{t}\left(h_{s}-h\right) .
$$

Here and in the following, vectors with the index $x y$ indicate the two-dimensional vector in the $x, y$ plane without the $z$ component. In addition, we have used the geometric relations

$$
\hat{\mathbf{n}}=\frac{\left(-\nabla_{x y} h, 1\right)}{\left[1+\left(\nabla_{x y} h\right)^{2}\right]^{1 / 2}},
$$




$$
v_{n}=\frac{\partial_{t} h}{\left[1+\left(\nabla_{x y} h\right)^{2}\right]^{1 / 2}},
$$

and we have defined the crystallization-dissolution rate along $z$ :

$$
v_{C z}=\partial_{t} h-u_{C z}+\mathbf{u}_{C x y} \cdot \nabla_{x y} h .
$$

Similarly, using Eqs. (8) and (9), mass conservation for the solute concentration $c$ can be rewritten in a two-dimensional form:

$$
\frac{v_{C z}}{\Omega}+\partial_{t}\langle c\rangle+\nabla_{x y} \cdot\left\langle\mathbf{u}_{L x y} c\right\rangle=-\nabla_{x y} \cdot\left\langle\mathbf{j}_{x y}\right\rangle .
$$

In order to write the force balance at the contact, we make use of two additional physical assumptions. First, we assume that the pressure outside the contact is approximately constant and equal to $p^{\text {ext }}$. Second, we assume that the interaction term vanishes, i.e., $W^{\prime} \approx 0$, away from the contact.

Finally, assuming that $\sigma^{\prime}$ vanishes outside the contact region force balance, Eq. (13), is rewritten as

$$
\mathbf{F}_{C}=\iint_{\text {contact }} d S\left[\hat{\mathbf{n}}\left(p-p^{e x t}+W^{\prime}(x, y, h)\right)-\hat{\mathbf{n}} \cdot \sigma^{\prime}\right] .
$$

This equation makes use of fact that the total force exerted by surface tension or by a constant external pressure on a crystal of arbitrary shape vanishes:

$$
\begin{gathered}
\oiint_{L C} d S \hat{\mathbf{n}}(\tilde{\gamma}: \kappa)=0, \\
\oiint_{L C} d S \hat{\mathbf{n}} p^{e x t}=0 .
\end{gathered}
$$

These two identities are proved in Appendix A.

\section{Lubrication limit in the contact region}

Here, we show that lubrication limit based on the small slope approximation allows one to express the quantities integrated along $z$ in Eqs. (14), (19), and (20), thereby leading to closedform equations for three quantities. The two first quantities are time- and space-dependent fields: the pressure $p$ and the thickness of the liquid film

$$
\zeta(x, y, t)=h_{s}(x, y)-h(x, y, t) .
$$

The third quantity is the time-dependent crystal velocity $\mathbf{u}_{C}$.

The lubrication limit [39] makes use of a disparity of length scales: the lateral extent of the film is assumed to be large $x \sim O\left(\epsilon^{-1}\right)$ as compared to the film thickness $\zeta=$ $\left(h_{s}-h\right) \sim O(1)$ with $\epsilon \ll 1$. The mathematical procedure to derive these equations is well known [34,39], and we therefore only provide the main steps of the derivation. Formally, we identify a small parameter $\epsilon=h_{0} / l$, where $l$ is the typical extent of the contact region and $h_{0}$ is the typical gap between the crystal and the substrate. Spatial coordinates then scale with this small parameter as $x \sim y \sim \ell \sim h_{0} / \epsilon$ and $z \sim h_{0}$. Furthermore, assuming that the typical fluid velocity parallel to the substrate is $\mathbf{u}_{L x y} \sim u_{0}$, we also consistently choose $u_{L z} \sim \epsilon u_{0}$, pressure $p \sim \eta u_{0} /\left(\epsilon h_{0}\right)$, and time $t \sim h_{0} / \epsilon u_{0}$.

Substituting these scalings of physical variables in the model equations, we obtain the lubrication expansion [34,39].
To leading order, Eq. (2) reduces to

$$
\begin{gathered}
\partial_{z} p=0, \\
-\nabla_{x y} p+\eta \partial_{z}^{2} \mathbf{u}_{L x y}=0 .
\end{gathered}
$$

The first equation indicates that the pressure does not depend on $z$, but only on $x, y$, and $t$. Solving the second equation using the boundary conditions Eqs. (4) and (5) results in a Poiseuille (parabolic) flow for $\mathbf{u}_{x y}$,

$$
\mathbf{u}_{L x y}=-\frac{\left(h_{s}-z\right)(z-h)}{2 \eta} \nabla_{x y} p+\frac{h_{s}-z}{\zeta} u_{C x y} .
$$

Integrating over the film thickness, we obtain

$$
\left\langle\mathbf{u}_{L x y}\right\rangle=-\frac{\zeta^{3}}{12 \eta} \nabla_{x y} p+\frac{\zeta}{2} \mathbf{u}_{C x y} .
$$

Combining Eqs. (15) and (26), we obtain

$$
\frac{\rho_{C}}{\rho_{L}} v_{C z}=\nabla_{x y} \cdot\left[\frac{\zeta^{3}}{12 \eta} \nabla_{x y} p-\mathbf{u}_{C x y} \frac{\zeta}{2}\right]-\partial_{t} \zeta .
$$

A similar procedure is applied to the concentration field. Assuming $c \sim O(1)$, we obtain to leading order from Eq. (6):

$$
\partial_{z}\left[D(c) \partial_{z} c\right]=0 .
$$

Integrating this relation and using local conservation of mass at the boundaries Eqs. (8) and (9), we obtain $\partial_{z} c=0$, showing that the concentration does not depend on $z$. Furthermore, assuming finite attachment-detachment kinetics $v \sim O(1)$ in Eq. (10) we obtain

$$
c=c_{e q}(x, y, t) \text {. }
$$

Hence for finite attachment-detachment kinetics, the concentration to leading order in the lubrication limit is equal to the local equilibrium concentration. This is the consequence of the smallness of the film thickness which enforces slow diffusion along the film, leaving ample time for local equilibration of the concentration via attachment and detachment of the LC interface. We may now write Eq. (19) using Sec. IIA in the lubrication limit as

$$
\begin{aligned}
& \frac{v_{C z}}{\Omega}+\partial_{t}\left[\zeta c_{e q}\right]-\nabla_{x y} \cdot\left[\frac{\zeta^{3}}{12 \eta} c_{e q} \nabla_{x y} p\right]+\frac{\mathbf{u}_{C x y}}{2} \cdot \nabla_{x y}\left[c_{e q} \zeta\right] \\
& =\nabla_{x y} \cdot\left[\zeta D\left(c_{e q}\right) \nabla_{x y} c_{e q}\right] .
\end{aligned}
$$

This relation involves $c_{e q}$, which depends on the chemical potential via Eq. (11). Let us compare the different contributions of the chemical potential. The lubrication expansion imposes $p \sim O\left(\epsilon^{-1}\right)$. For disjoining forces to be able to balance viscous forces, we choose $W^{\prime}(x, y, h) \sim O\left(\epsilon^{-1}\right)$. As a consequence, the pressure term and the interaction term in Eq. (12) are of the same order of magnitude. In addition, since the curvature $\kappa \sim \partial_{x x} h \sim \partial_{y y} h \sim \epsilon^{2}$ is small, only large stiffnesses $\tilde{\gamma} \sim O\left(\epsilon^{-3}\right)$ can make the capillary term $\tilde{\gamma}: \kappa$ relevant. However even if surface stiffness is not so large, the capillary term can be relevant in two cases: (i) if the curvature locally blows up and (ii) far from the substrate where the potential term $W^{\prime}$ can be neglected. We will see in the following that these conditions can be reached during pressure 
solution. Therefore, in order to include all relevant cases for the discussion below, we keep the capillary term leading to

$$
\begin{aligned}
\frac{\Delta \mu(x, y, t)}{\Omega}= & -\tilde{\gamma}_{1} \partial_{x_{1} x_{1}} h-\tilde{\gamma}_{2} \partial_{x_{2} x_{2}} h \\
& +W^{\prime}(x, y, h)+\left(\frac{\rho_{C}}{\rho_{L}}-1\right) p,
\end{aligned}
$$

where $x_{1}$ and $x_{2}$ are the directions of principal curvature of the LC interface, and $\tilde{\gamma}_{1}, \tilde{\gamma}_{2}$ are the related surface stiffnesses [40].

Finally, since $W^{\prime}$ is of the same order as $p$ in the lubrication limit, force balance Eq. (20) reads

$$
\begin{gathered}
F_{C z}=\iint_{\text {contact }} d A\left(p-p^{e x t}+W^{\prime}(x, y, h)\right), \\
\mathbf{F}_{C x y}=\iint_{\text {contact }} d A\left(\frac{\eta \mathbf{u}_{C x y}}{\zeta}-\left(p-p^{e x t}\right) \nabla_{x y}\left(h_{s}-\frac{\zeta}{2}\right)\right),
\end{gathered}
$$

where $d A=d x d y$. To derive the last relation, we have assumed that, at the boundary of the contact zone, $p=p^{e x t}$ is constant and $\zeta$ is large enough for $W^{\prime}$ to be negligible.

As a summary, we have derived a thin film model for the contact region during dissolution and growth, which consists of two equations, Eqs. (27) and (30), for the coupled twodimensional space and time-dependent fields $p$ and $\zeta$, and an additional vectorial integral constraint, Eqs. (32) and (33), which determines the time-dependent crystal velocity $\mathbf{u}_{C}$. This system is not only nonlinear but also nonlocal due to the force balance equation. In the following, we explore some consequences of the model in the specific case of pressure solution of a single contact.

\section{Ridge and axisymmetric contact}

We now consider the pressure solution of a single contact with some simplifying assumptions:

(i) equal densities between the liquid and the crystal $\rho_{C}=$ $\rho_{L}$; 0 ;

(ii) no lateral motion $\mathbf{u}_{C x y}=0$ and no lateral force $F_{C x y}=$

(iii) diffusion constant independent of concentration $D(c)=D$;

(iv) isotropic surface tension $\tilde{\gamma}_{1}=\tilde{\gamma}_{2}=\gamma$;

(v) flat substrate $h_{s}$ independent of $x$ and $y$. We use the interaction potential $U$, defined by $U(\zeta)=W(x, y, h)$, and it follows that $W^{\prime}(h)=\partial_{h} W(h)=-\partial_{\zeta} U(\zeta)=-U^{\prime}(\zeta)$;

(vi) small concentrations $\Omega c_{e q} \ll 1$;

(vii) linearized Gibbs-Thomson relation $\Delta \mu / k_{B} T \ll 1$.

In addition, we consider two simple geometries. The first one is a one-dimensional ridge, which is invariant along $y$, and left-right symmetric with $h(x)=h(-x)$. The second geometry is an axisymmetric contact, the shape of which depends only on the distance $r$ from the origin in the $x, y$ plane. In the following, we will often refer to the symmetric ridge as 1D and the axisymmetric contact as 2D.

\section{Symmetric ridge}

Consider first the ridge case obeying the $x \rightarrow-x$ symmetry, with a system length $2 L$. Assuming $\rho_{C}=\rho_{L}$, the integration of Eq. (27) leads to

$$
p=p^{e x t}+u_{C z} \int_{x}^{L} d x \frac{12 \eta x}{\zeta^{3}} .
$$

Plugging this expression into Eq. (32) provides us with a nonlocal relation between the crystal velocity and the surface height:

$$
2 u_{C z} \int_{0}^{L} d x \int_{x}^{L} d x^{\prime} \frac{12 \eta x^{\prime}}{\zeta^{3}}=F_{C z}^{1 D}+2 \int_{0}^{L} d x U^{\prime}(\zeta) .
$$

This equation relates the sum of the load and interaction forces between the crystal and the substrate on the right-hand side to the forces caused by viscous dissipation in the film on the left-hand side. In the viscous term, the crystal velocity $u_{C z}$ is multiplied by the hydrodynamic mobility of the crystal which depends on the interface profile $\zeta$. The expression of this mobility is well known in the lubrication limit [30].

In the limit of small concentrations $\Omega c_{e q} \ll 1$ and equal densities $\rho_{L}=\rho_{C}$, Eq. (30) takes a simple form:

$$
\partial_{t} \zeta=-D \Omega \partial_{x}\left[\zeta \partial_{x} c_{e q}\right]-u_{C z} .
$$

Assuming that $\Delta \mu / k_{B} T \ll 1$ in Eq. (11) and using Eq. (31), we obtain

$$
\partial_{t} \zeta=-D_{e} \partial_{x}\left\{\zeta \partial_{x}\left[\gamma \partial_{x x} \zeta-U^{\prime}(\zeta)\right]\right\}-u_{C z},
$$

where by definition

$$
D_{e}=\frac{D \Omega^{2} c_{0}}{k_{B} T} .
$$

\section{Axisymmetric contact}

Let us now consider an axisymmetric contact. Using cylindrical coordinates in a contact zone of radius $R$, we obtain in a similar way the following equations:

$$
\begin{gathered}
2 u_{C z} \pi \int_{0}^{R} d r r \int_{r}^{R} d r^{\prime} \frac{6 \eta r^{\prime}}{\zeta\left(r^{\prime}\right)^{3}}=F_{C z}^{2 D}+2 \pi \int_{0}^{R} d r r U^{\prime}(\zeta), \\
\partial_{t} \zeta=-D_{e} \frac{1}{r} \partial_{r}\left\{r \zeta \partial_{r}\left[\gamma \partial_{r r} \zeta+\frac{\gamma}{r} \partial_{r} \zeta-U^{\prime}(\zeta)\right]\right\}-u_{C z},
\end{gathered}
$$

where the quantity proportional to $\gamma$ is the mean curvature in axial symmetry $[42,43]$.

\section{Interaction potentials}

We chose to study two generic types of repulsive interaction potentials. The first one diverges when the film thickness $\zeta$ vanishes,

$$
U(\zeta)=\frac{A}{\zeta^{n}},
$$

where $A$ is a constant. In practice, numerical results have been obtained with $n=3$. However, we will keep an arbitrary exponent $n$ in the discussions.

The second type of potential exhibits a finite repulsion when $\zeta \rightarrow 0$

$$
U(\zeta)=A e^{-\frac{\zeta}{\lambda}}
$$


where $\lambda$ is a decay length representing for instance the Debye length in the case of electrostatic interactions [19].

The essential difference between these potentials is that Eq. (41) leads to an infinite repulsion force when $\zeta \rightarrow 0$, whereas this force is finite for Eq. (42).

\section{METHODS}

\section{A. Normalization}

In order to perform simulations and to analyze the results of the model, we write the model equations in a dimensionless form and identify the relevant dimensionless parameters. All variables appearing in normalized units are labeled with a top bar.

We start by defining the dimensionless repulsion strength $\bar{A}$. For the exponential potential we set $\bar{A}=A / \gamma$, while for power-law repulsions with the case $n=3$, we use $\bar{A}=$ $A /\left(\gamma \lambda^{3}\right)$. The normalized film thickness is $\bar{\zeta}=\zeta / \lambda$, and the normalized coordinates are $\bar{x}=x \bar{A}^{1 / 2} / \lambda, \bar{y}=y \bar{A}^{1 / 2} / \lambda$. The normalized time is defined as $\bar{t}=t D_{e} \gamma \bar{A}^{2} / \lambda^{3}$. The normalized equations are showed in Appendix B. Notice that the scale $\lambda$ is imposed by the expression of $U$ in the case of an exponential repulsion, while it is an arbitrary length scale corresponding to the actual film width in the case of power-law repulsions.

The normalized repulsion strength $\bar{A}$ comes into play in spatiotemporal scales but not as a parameter of the normalized equations. As a consequence, it cannot change the model behavior qualitatively. The only parameters explicitly appearing in the normalized equations are the normalized viscosity $\bar{\eta}$ and external load $\bar{F}_{C z}$. The normalized viscosity reads

$$
\bar{\eta}=\frac{D_{e}}{\lambda^{2}} \eta=\frac{D \Omega^{2} c_{0}}{\lambda^{2} k_{B} T} \eta .
$$

Since the loads have different dimensionality in 1D (force per unit length) and 2D (force), their normalization is different:

$$
\bar{F}_{C z}^{1 D}=\frac{F_{C z}^{1 D}}{\gamma \bar{A}^{1 / 2}}, \quad \bar{F}_{C z}^{2 D}=\frac{F_{C z}^{2 D}}{\gamma \lambda} .
$$

Below, all simulations are performed with normalized variables and coordinates. However, the analysis of the equations is performed in physical coordinates to make the physical interpretation more transparent.

\section{B. Numerical methods}

We solved Eqs. (35) and (37) or Eqs. (39) and (40) using an explicit Euler method, where derivatives are calculated with the help of a finite difference scheme. We imposed a fixed interface height at the boundary of the contact region, $\zeta=\zeta_{b c}$, where $x= \pm L$ or $r=R$. The gap $\zeta_{b c}$ between the crystal and the substrate at the boundary is chosen to be large as compared to the range of the interaction potential, but small as compared to the contact region width $L$, or $R$. We also impose a constant supersaturation at the boundary $\Delta C=c_{e q} / c_{0}-1 \approx$ $\Delta \mu /\left(k_{B} T\right)$ to mimic a macroscopic concentration bath outside the contact.

The boundary conditions introduce three additional dimensionless parameters: the normalized system size

$$
\bar{L}=\frac{L \bar{A}^{1 / 2}}{\lambda} \text { or } \quad \bar{R}=\frac{R \bar{A}^{1 / 2}}{\lambda},
$$

the normalized film thickness at the boundary

$$
\bar{\zeta}_{b c}=\frac{\zeta_{b c}}{\lambda}
$$

and the normalized supersaturation

$$
\overline{\Delta C}=\frac{k_{B} T \lambda}{\bar{A} \gamma \Omega} \Delta C
$$

Simulations are performed with $\bar{L}, \bar{R}=100$, substrate position $\bar{h}_{s}=2$, film thickness at the boundary $\bar{\zeta}_{b c}=12$, and boundary supersaturation $\Delta C=0$. The discretization bin size is $\Delta \bar{x}=0.2$ for most simulations. However, in some cases, to be able to resolve the contact shape at very high external forces (see Sec. IV B and Fig. 7), it was necessary to increase the spatial resolution up to 16 times.

The simulations were always started with a flat profile (see top panel of Fig. 3). When applying a concentration higher than the equilibrium one at the boundary, we observe crystal growth: The crystal translates downward by addition of growth units at the surface, and $u_{C z}<0$. When applying an external load, $F_{C z}$, with sign in the positive direction, hence pushing the crystal toward the substrate, we observe dissolution, i.e., pressure solution and $u_{C z}>0$. The latter case is the main focus of this paper.

\section{RESULTS: SINGLE CONTACT PRESSURE SOLUTION}

As an illustrative example, we show in Fig. 3 the numerical solution for the profile of a ridge obeying Eqs. (35) and (37) when an external load pushes the crystal upward against the substrate and when the interaction is in the form of a singular repulsion, Eq. (41). A similar shape is observed when solving Eqs. (39) and (40) for an axisymmetric contact looking at the section along the radius. The simulation shows that the interface profile reaches a steady state characterized by a constant crystal velocity (dissolution rate) and fixed interface position.

As discussed earlier in Sec. IIC, in the contact region and in the absence of blowup of the curvature, we expect the surface tension contribution to be small. Neglecting this contribution, steady-state solutions with a constant profile, i.e., $\partial_{t} \zeta=0$, obey respectively in $1 \mathrm{D}$ or $2 \mathrm{D}$

$$
\begin{aligned}
& 0=u_{c z}-D_{e} \partial_{x}\left[\zeta \partial_{x} U^{\prime}(\zeta)\right], \\
& 0=u_{c z}-\frac{D_{e}}{r} \partial_{r}\left[r \zeta \partial_{r} U^{\prime}(\zeta)\right] .
\end{aligned}
$$

This equation is integrated as

$$
\begin{aligned}
\frac{x^{2}}{2 D_{e}} u_{C z} & =\tilde{U}(\zeta(r))-\tilde{U}\left(\zeta_{0}\right), \\
\frac{r^{2}}{4 D_{e}} u_{C z} & =\tilde{U}(\zeta(r))-\tilde{U}\left(\zeta_{0}\right),
\end{aligned}
$$



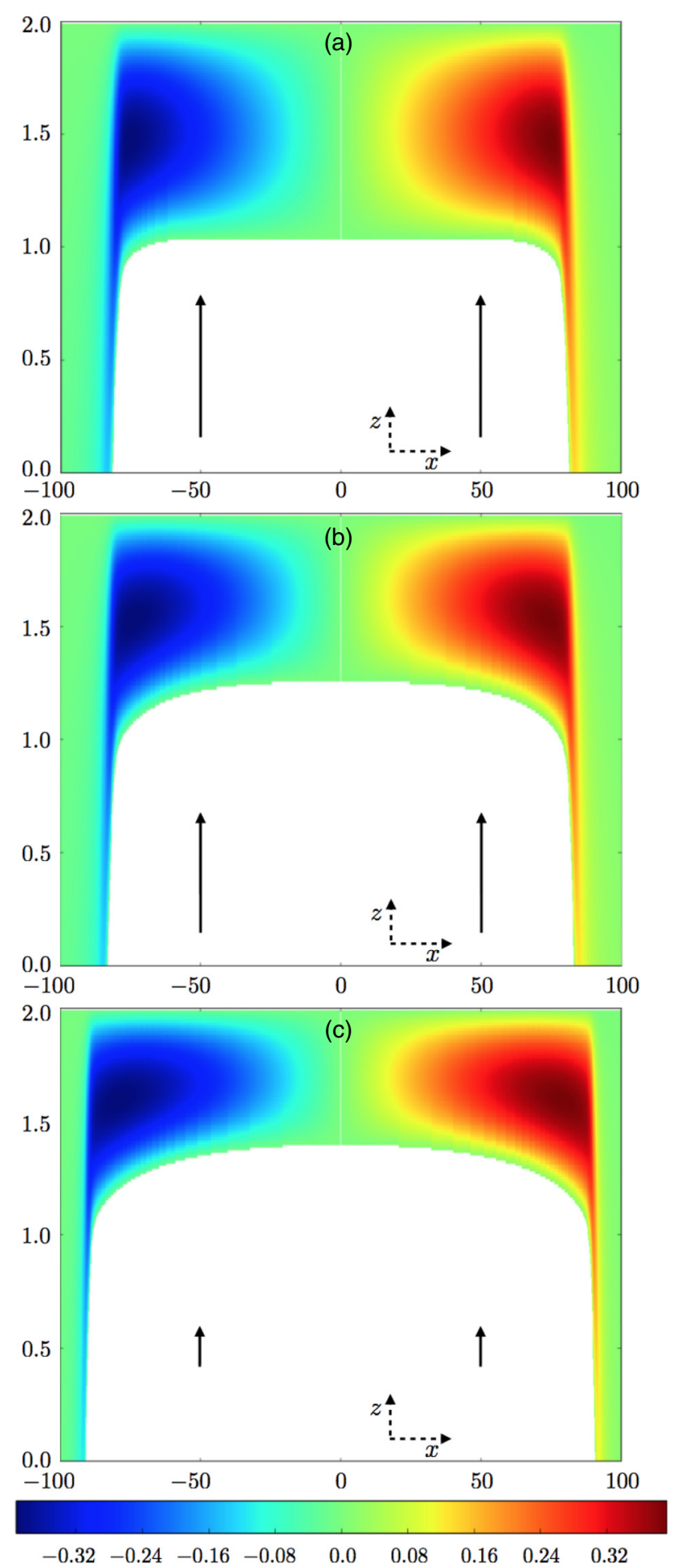

FIG. 3. Pressure solution dynamics. Snapshots of the numerical solution of Eq. (37) representing a dissolving contact ridge. Size of the simulation box $\bar{L}=100$ (physical size $L \approx 1 \mu \mathrm{m}$ ) under an external pressure $p=26 \mathrm{MPa}$. The viscosity is $\bar{\eta}=0.5$. The crystal is in white, and the black arrows are proportional to the crystal velocity $u_{C z}$. The time increases from the top panel to the bottom one. (a) Initial condition. As an example, using physical constants related to calcite (see Sec. VB), physical time frames are (b) $10 \mathrm{~s}$ and (c) $6.7 \mathrm{~min}$. The color map (in arbitrary units) shows the amplitude of the $x$ component of liquid velocity field $\mathbf{u}_{L x y}$, as obtained from Eqs. (25) and (34). The vertical scale is in nanometers. The substrate is located at $h_{s}=2 \mathrm{~nm}$. where $\zeta_{0}=\zeta(0)$, and $\tilde{U}(\zeta)$ is defined via the relation

$$
\tilde{U}^{\prime}(\zeta)=\zeta U^{\prime \prime}(\zeta)
$$

which, up to an additive constant leads to $\tilde{U}(\zeta)=\zeta U^{\prime}(\zeta)-$ $U(\zeta)$. Since we expect physically that the interaction potential tends to a constant as $\zeta \rightarrow \infty$, i.e., that $U(\infty)$ is a constant, then $\tilde{U}(\infty)$ should also be a constant. Therefore, $\tilde{U}$ cannot increase indefinitely when $\zeta \rightarrow \infty$ on the right-hand side of Eqs. (47a) and (47b). As a consequence, there are finite $x_{m}$ or $r_{m}$ where $\zeta \rightarrow \infty$ and they obey

$$
\begin{aligned}
\frac{x_{m}^{2}}{2 D_{e}} u_{C z} & =\tilde{U}(\infty)-\tilde{U}\left(\zeta_{0}\right), \\
\frac{r_{m}^{2}}{4 D_{e}} u_{C z} & =\tilde{U}(\infty)-\tilde{U}\left(\zeta_{0}\right) .
\end{aligned}
$$

Since $\zeta$ diverges at some finite distance $x_{m}$ or $r_{m}$ from the center of the contact, the size of the contact in steady-state pressure solution is always finite.

In the limit of large forces, we expect $\zeta_{0}$ to become small. The situation then turns out to be very different depending on how $\tilde{U}\left(\zeta_{0}\right)$ behaves when $\zeta_{0}$ is small. The following sections discuss separately the cases of finite and diverging interaction potentials $U(\zeta)$, corresponding to finite or diverging $\tilde{U}(\zeta)$ as $\zeta \rightarrow 0$.

\section{A. Singular repulsion: Power-law case}

Let us start with the analysis of the results for a singular power-law repulsion between the crystal surface and the substrate. Combining Eqs. (41) and (48), we find

$$
\tilde{U}(\zeta)=\frac{-(n+1) A}{\zeta^{n}} .
$$

Inserting this expression in Eqs. (47a) and (47b) provides us with the steady-state profile:

$$
\begin{aligned}
& \zeta(x)=\left(\frac{\zeta_{0}^{n}}{1-x^{2} / x_{m}^{2}}\right)^{1 / n}, \\
& \zeta(r)=\left(\frac{\zeta_{0}^{n}}{1-r^{2} / r_{m}^{2}}\right)^{1 / n} .
\end{aligned}
$$

These profiles diverge at $x=x_{m}$ or $r=r_{m}$, which is related to the minimum distance in the contact via Eqs. (49a) and (49b):

$$
\begin{aligned}
x_{m}^{2} & =\frac{2 D_{e}(n+1) A}{\zeta_{0}^{n} u_{C z}}, \\
r_{m}^{2} & =\frac{4 D_{e}(n+1) A}{\zeta_{0}^{n} u_{C z}} .
\end{aligned}
$$

The distance $x_{m}$ or $r_{m}$ at which the profile diverges should $a$ priori be distinguished from the size of the contact region. Indeed, far away from the substrate, the influence of the potential vanishes, and as a consequence surface-tension effects should become dominant, so that Eq. (47) is not valid anymore. Let us define $L_{c}$ as the half-width of the contact region in 1D, and $R_{c}$ as the radius of the contact region in $2 \mathrm{D}$. An intuitive definition of the contact region is the zone which is close enough to the substrate to be under the influence of the interaction potential $U$. 


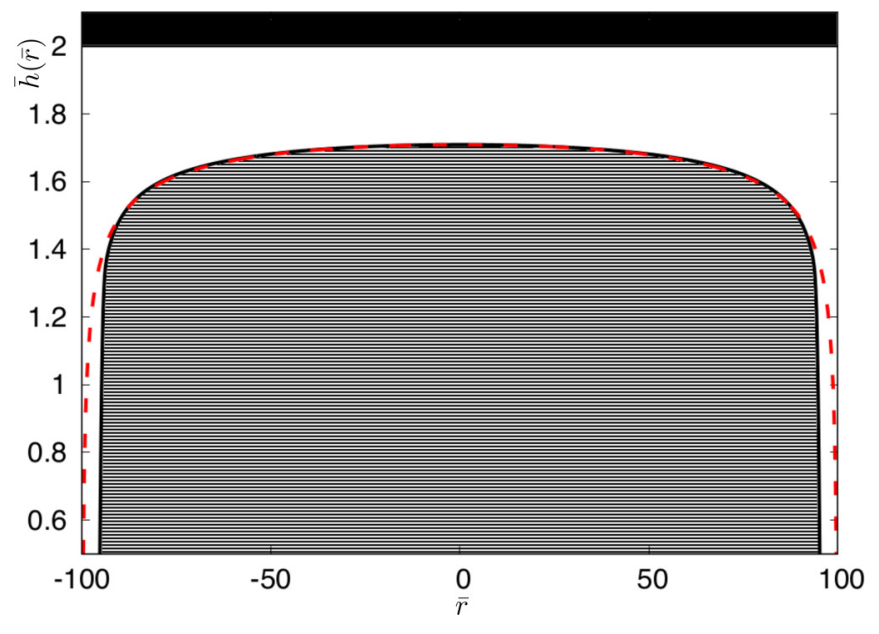

FIG. 4. Flattened steady-state for power-law repulsion. Cross section of the steady-state profile projected along $\bar{r}$ (solid line) dissolving under an external load, $\bar{F}_{C z}=10^{8}$, at $\bar{\eta}=1$ against a flat substrate $\left(\bar{h}_{s}=2\right)$. Geometry: axisymmetric contact in a simulation box of size $\bar{R}=100$. The interaction with the substrate is a singular power-law repulsion, Eq. (41) with $n=3$. The red dashed line is the analytical prediction from Eq. (51) with $r_{m}=R$ and $\zeta_{0} \approx 0.29$ as a fitting parameter.

For large contacts, we expect that the distance separating $x_{m}$ and $L_{c}$, or $r_{m}$ and $R_{c}$ should be negligible as compared to the size of the contact region. As a consequence, we assume $x_{m} \approx L_{c}$ or $r_{m} \approx R_{c}$. Furthermore, we perform simulations with a fixed $\zeta_{b c}$, which is large as compared to $\zeta_{0}$ but small as compared to the size $L$, or $R$ of the simulation box. Thus, the contact region should fill most of the simulation box, and finally we expect $x_{m} \approx L_{c} \approx L$ or $r_{m} \approx R_{c} \approx R$. In Fig. 4, we show the steady-state cross section obtained from the simulation (solid line) at large times, which is in good agreement with Eq. (51) using $r_{m}=R$ (dashed line) and $\zeta_{0}$ as a fitting parameter. Using Eq. (52) and the fitted value of $\zeta_{0}$, we obtain a value for $u_{C z}$. For instance, in $2 \mathrm{D}$ with $\bar{F}=10^{8}$ and $\bar{R}=100$, this procedure leads to $\bar{\zeta}_{0}=0.290$ and $\bar{u}_{C z}=0.022$ being compared with $\bar{\zeta}_{0}=0.291$ and $\bar{u}_{C z}=0.016$ measured directly in the numerical solution of the full model. The agreement with the numerical results improves as the external load is increased.

Similar agreement is obtained in 1D. As a consequence, the profile is well predicted at large forces, and we can safely use it in the force balance equation.

In 1D, using Eq. (51) with Eq. (52a) and $x_{m}=L_{c}$, we obtain from force balance Eq. (35)

$$
\begin{aligned}
\frac{F_{C z}^{1 D}}{L_{c}}= & 24 \eta \phi\left(\frac{n+3}{n}\right) \frac{n \sqrt{\pi}}{(n+3)}\left[\frac{1}{D_{e} A(n+1)}\right]^{\frac{3}{n}}\left(\frac{L_{c}^{2}}{2} u_{C z}\right)^{\frac{n+3}{n}} \\
& +\phi\left(\frac{n+1}{n}\right) 2 n \sqrt{\pi} A^{-\frac{1}{n}}\left(\frac{1}{D_{e}(n+1)}\right)^{\frac{n+1}{n}} \\
& \times\left(\frac{L_{c}^{2}}{2} u_{C z}\right)^{\frac{n+1}{n}}
\end{aligned}
$$

where

$$
\phi(z)=\frac{\Gamma(1+z)}{2 \Gamma\left(\frac{3}{2}+z\right)},
$$

with $\Gamma$ being the Euler $\Gamma$ function.

Similarly, in 2D force balance, Eq. (39) imposes

$$
\begin{aligned}
\frac{F_{C z}^{2 D}}{\pi R_{c}^{2}}= & 12 \eta \frac{n^{2}}{(2 n+3)(n+3)}\left[\frac{1}{D_{e} A(n+1)}\right]^{\frac{3}{n}}\left(\frac{R_{c}^{2}}{4} u_{C z}\right)^{\frac{n+3}{n}} \\
& +\frac{n^{2}}{2 n+1} A^{-\frac{1}{n}}\left[\frac{1}{D_{e}(n+1)}\right]^{\frac{n+1}{n}}\left(\frac{R_{c}^{2}}{4} u_{C z}\right)^{\frac{n+1}{n}}
\end{aligned}
$$

(some technical details about the derivation of this relation can be found in Appendix C 1). Using Eq. (54), we find two separate regimes depending on the value of $\eta$ : For large viscosities we identify a hydrodynamic regime

$$
\begin{aligned}
& u_{C z}^{1 D}=C_{h}^{1 D} L_{c}^{-\frac{3 n+6}{n+3}}\left(\frac{F_{C z}^{1 D}}{\eta}\right)^{\frac{n}{n+3}}, \\
& u_{C z}^{2 D}=C_{h}^{2 D} R_{c}^{-\frac{4 n+6}{n+3}}\left(\frac{F_{C z}^{2 D}}{\eta}\right)^{\frac{n}{n+3}},
\end{aligned}
$$

while for small viscosities a diffusion regime is found, with

$$
\begin{aligned}
& u_{C z}^{1 D}=C_{d}^{1 D} L_{c}^{-\frac{3 n+2}{n+1}}\left(F_{C z}^{1 D}\right)^{\frac{n}{n+1}}, \\
& u_{C z}^{2 D}=C_{d}^{2 D} R_{c}^{-\frac{4 n+2}{n+1}}\left(F_{C z}^{2 D}\right)^{\frac{n}{n+1}} .
\end{aligned}
$$

The expressions of the constants $C_{h}^{1 D}, C_{h}^{2 D}, C_{d}^{1 D}, C_{d}^{2 D}$ are reported in Appendix C 1. In Fig. 5, we compare the prediction of Eqs. (55b) and (56b) using $R_{c}=R$ (solid and dashed lines) and the results in $2 \mathrm{D}$ obtained from the complete numerical solution of the model (circles). The analytical prediction is in good agreement with the numerical solution for large external loads.

In order to probe the sensitivity of the results with respect to the value of the film thickness at the boundary $\bar{\zeta}_{b c}$, we monitored the consequences of the variation of $\bar{\zeta}_{b c}$. We found small quantitative effects but no influence on the qualitative behavior of the relevant observables. This is exemplified with the variations of the dissolution rates in the top panel of Fig. 5.

Using Eq. (52) to eliminate $u_{C z}$ in the expression of the force Eqs. (53) and (54), a relation between external load and the minimum thickness $\zeta_{0}$ can be obtained, which is found to be in good agreement with the simulations. For the sake of concision, the expression of this relation in 2D and its comparison with the numerical solution of the full model are shown in Appendix C (Eq. (C8) and Fig. 9).

As an additional remark, Eqs. (53) and (54) show that there is no substantial difference between one and two dimensions except, as expected from dimensional analysis, a different scaling with the contact size. Finally, it is interesting to assess the critical length and load separating the diffusive and hydrodynamic regimes. Equating the expressions of the velocity in the two regimes for the ridge case, we find that the critical size above which the force is dominated by the diffusion term is given by

$$
L_{*}=B_{1 D} \eta^{\frac{n+1}{2}} F_{C z}^{1 D}
$$



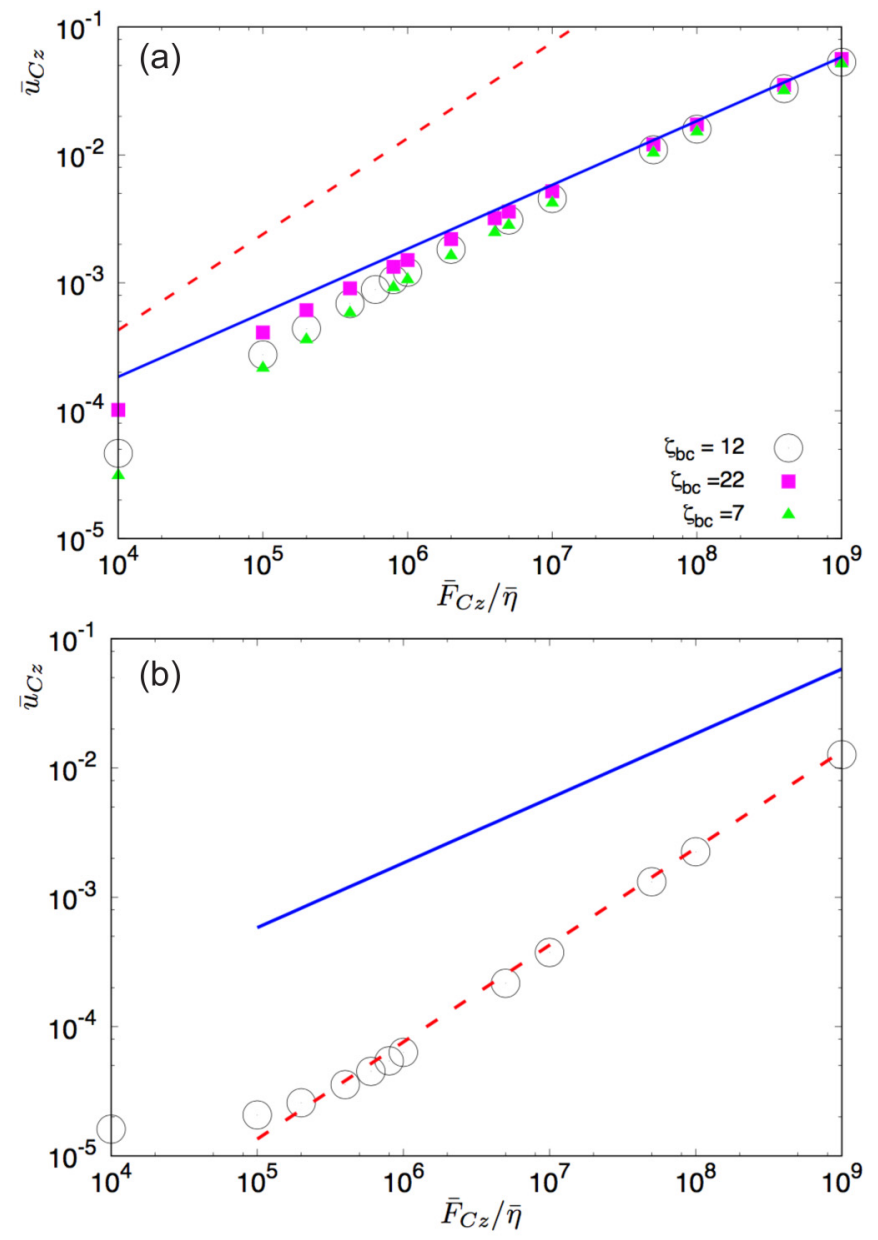

FIG. 5. Dissolution rate for (singular) power-law repulsion $(n=$ 3 ) as a function of the applied load. Geometry: axisymmetric contact. Lines represent asymptotic analytical predictions with $\bar{R}_{c}=\bar{R}=$ 100: Solid blue line, hydrodynamic regime Eq. (55b); dashed red line, diffusion regime Eq. (56b). (a); the symbols show simulation results obtained using different boundary thickness $\zeta_{b c}$. (b) $\bar{\eta}=10^{-3}$. The results are shown in normalized units.

in $1 \mathrm{D}$, while for the axisymmetric contact is

$$
R_{*}=B_{2 D} \eta^{\frac{n+1}{4}}\left(F_{C z}^{2 D}\right)^{1 / 2},
$$

where $B_{1 D}$ and $B_{2 D}$ are constants reported in Appendix C1. Hence, at fixed force, large contacts will be dominated by the diffusion term. Also, as the external load is increased at constant contact size, the hydrodynamic term in the force balance become dominant. Once again, good agreement with the simulations is found, and a detailed discussion is reported in Appendix C1.

\section{B. Finite repulsion: Exponential case}

In the case of an exponential repulsion, $\tilde{U}(\zeta=0)$ is finite. As a consequence, the behavior of steady-state solutions is different. First, the dissolution rate is asymptotically independent of the load. Second, the shape of the contact is sharp and pointy. Third, in the absence of surface tension, touching contact (i.e., $\zeta=0$ ) would be observed in 2D for a finite loading force, but not in 1D. Finally, as opposed to what observed so far, surface

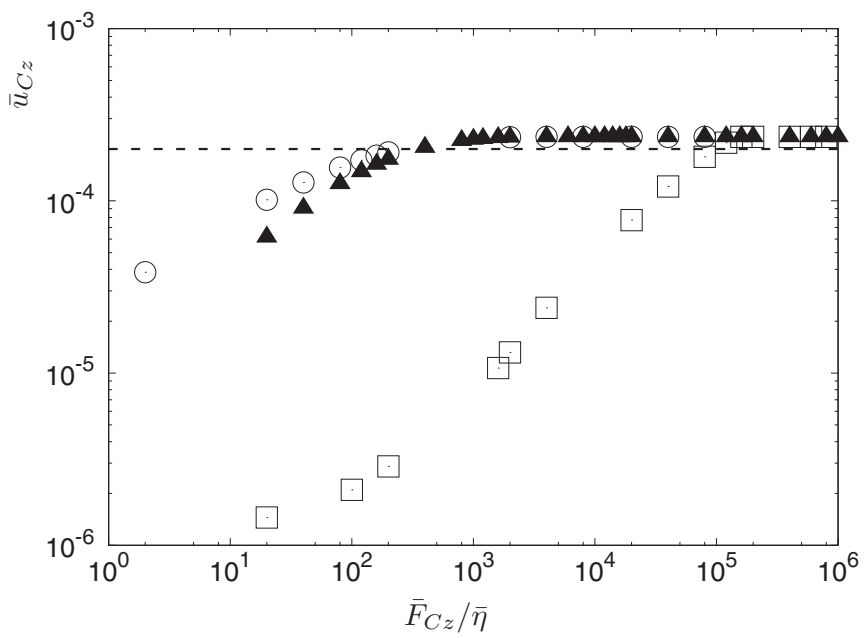

FIG. 6. Dissolution rate as a function of the external load for an exponential finite interaction. Geometry: 1D ridge contact. Triangles $\bar{\eta}=0.5$, squares $\bar{\eta}=5 \times 10^{-4}$, circles $\bar{\eta}=5 \times 10^{2}$. Dashed line: analytical prediction Eq. (61a) using $\bar{L}_{c}=\bar{L}=100$ and $\zeta_{0} / \lambda=0$. The results are in normalized units.

tension becomes relevant at large enough forces both in $1 \mathrm{D}$ and $2 \mathrm{D}$, and prevents contact also in 2D.

\section{Without surface tension}

Neglecting surface tension, we proceed in a similar way as in the power-law case. Recalling Eq. (48) and using the exponential interaction potential Eq. (42), we now have

$$
\tilde{U}(\zeta)=-\frac{A}{\lambda}(\lambda+\zeta) e^{-\frac{\zeta}{\lambda}}
$$

As opposed to the power-law repulsion case, we now have a function $\tilde{U}(\zeta)$ that cannot be inverted explicitly. Therefore, $\zeta$ cannot be explicitly obtained from Eq. (47). However, since $\tilde{U}$ is a monotonic function of $\zeta$, it is still possible to compute $r$ as a function of $\zeta$ without ambiguity from Eq. (47).

In the large force limit, since we expect $\zeta_{0} \ll \lambda$ (this will be confirmed below using force balance) and since $\tilde{U}(0)$ is finite, we find that the dissolution rate reaches a constant value independent of the load and of the viscosity. Indeed, from Eq. (49),

$$
\begin{aligned}
& u_{C z}^{1 D} \approx D_{e} \frac{2 A}{L_{c}^{2}}\left(1+\frac{\zeta_{0}}{\lambda}\right) e^{-\frac{\zeta_{0}}{\lambda}}, \\
& u_{C z}^{2 D} \approx D_{e} \frac{4 A}{R_{c}^{2}}\left(1+\frac{\zeta_{0}}{\lambda}\right) e^{-\frac{\zeta_{0}}{\lambda}} .
\end{aligned}
$$

Taking the limit $\zeta_{0} \rightarrow 0$, we find

$$
\begin{aligned}
& u_{C z}^{1 D}=D_{e} \frac{2 A}{L_{c}^{2}}, \\
& u_{C z}^{2 D}=D_{e} \frac{4 A}{R_{c}^{2}} .
\end{aligned}
$$

Again assuming that $L_{c} \approx L$, or $R_{c} \approx R$ at large forces, these results are confirmed in Fig. 6 from the comparison with the numerical solution of the full model. The different viscosities, indicated by circles $(\bar{\eta}=1000)$, triangles $(\bar{\eta}=1)$, and squares 


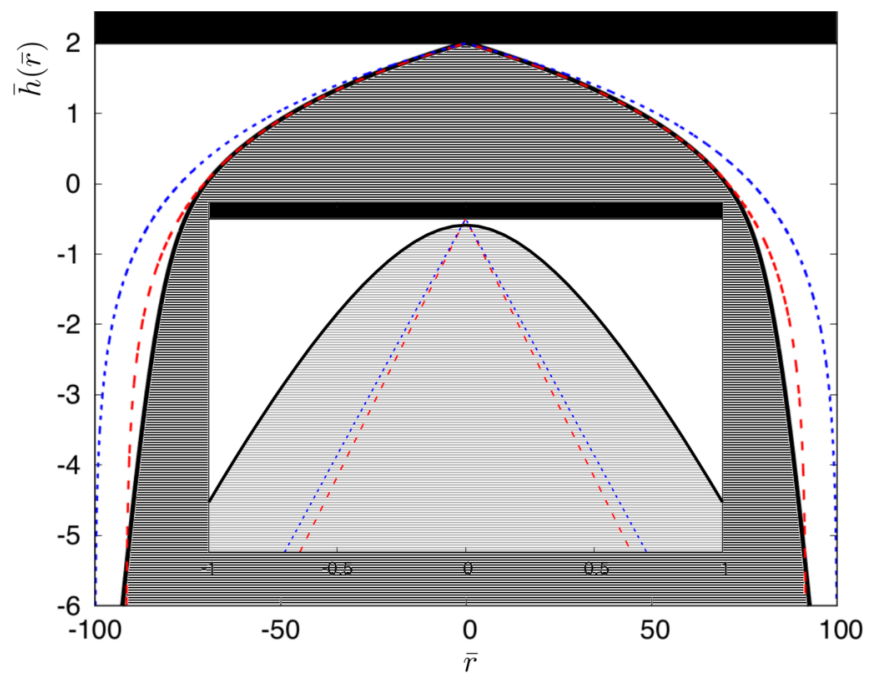

FIG. 7. Pointy steady state for exponential repulsion. Cross section of the steady-state profile projected along $\bar{r}$ (solid line) dissolving under an external load $\bar{F}_{C z}=1.7 \times 10^{5}$, at $\bar{\eta}=1$ against a flat substrate $\left(\bar{h}_{s}=2\right)$. Geometry: axisymmetric contact in a simulation box of size $\bar{R}=100$. The interaction with the substrate is a finite exponential repulsion, Eq. (42). Blue dotted line: analytical prediction Eq. (40), assuming the contact area to be equal to the surface size $R$. Red dashed line: analytical prediction Eq. (40) with a smaller contact size $R_{c}$. The inner plot shows a zoom of the tip.

( $\bar{\eta}=0.001)$, affect the absolute value of the applied force needed to reach the plateau but not the plateau value itself.

A second consequence arising from the finiteness of the exponential interaction is the sharp pointy shape of the steadystate profile showed in Fig. 7. Indeed, since $\tilde{U}^{\prime}(\zeta=0)=0$ from Eq. (48), we have $\tilde{U}(\zeta) \approx \tilde{U}(0)+\tilde{U}^{\prime \prime}(0) \zeta^{2} / 2$ for $\zeta \ll \lambda$. Using this expansion into Eq. (47) and letting $\zeta_{0} \rightarrow 0$, we find that the profile $\zeta_{\text {sing }}$ in the center of the contact region is a singular wedge in $1 \mathrm{D}$ and a cone in $2 \mathrm{D}$ :

$$
\begin{gathered}
\zeta_{\text {sing }} \approx\left(\frac{u_{C z}}{D_{e} \tilde{U}^{\prime \prime}(0)}\right)^{1 / 2}|x|=\left(\frac{u_{C z}}{D_{e} A}\right)^{1 / 2} \lambda|x|, \\
\zeta_{\text {sing }} \approx\left(\frac{u_{C z}}{2 D_{e} \tilde{U}^{\prime \prime}(0)}\right)^{1 / 2}|r|=\left(\frac{u_{C z}}{2 D_{e} A}\right)^{1 / 2} \lambda|r| .
\end{gathered}
$$

When $\zeta_{0} \ll \lambda$, the complete profile for arbitrary $\zeta$ (i.e., smaller or larger than $\lambda$ ) can be obtained from Eqs. (47) and (59). Using the axisymmetric contact, with $R_{c}=R$ and $u_{C z}$ given by Eq. (61b), this expression (dotted blue line) is seen to be in good agreement with the simulation in Fig. 7. Better agreement (red dashed line) can be reached using the numerical value of $u_{C z}$ obtained from the simulation (which is equivalent to assuming a smaller effective size, $R_{c}<R$ ). Nevertheless, as shown by the inner panel in Fig. 7, close to the tip the numerical solution is smoothed and exhibits a parabolic shape. This regularization discussed in the next section is due to the contribution of the surface tension.

Using Eq. (35), force balance in 1D now reads

$$
\frac{F_{C z}^{1 D}}{L_{c}}=\left[12 \eta D_{e} \frac{A}{\lambda^{3}} \psi_{1}\left(\frac{\zeta_{0}}{\lambda}\right)+\frac{A}{\lambda} \psi_{2}\left(\frac{\zeta_{0}}{\lambda}\right)\right]\left(\frac{e^{\frac{\zeta_{0}}{\lambda}}}{1+\frac{\zeta_{0}}{\lambda}}\right)^{\frac{1}{2}}
$$

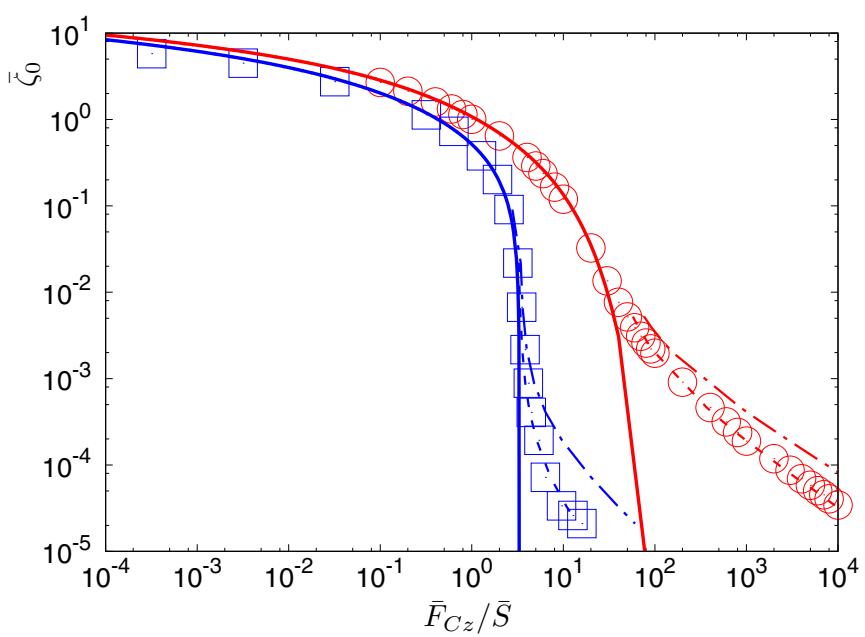

FIG. 8. Minimum film thickness of the liquid film as a function of the applied load. The plot shows the minimum distance $\bar{\zeta}_{0}$ between the crystal and the substrate versus the external load normalized by surface area $\bar{S}$ (scaled pressure). Red, ridge contact (1D); blue, axisymmetric contact (2D). Circles (1D) and squares (2D) show the numerical results. Solid lines report the analytical predictions neglecting surface tension, Eqs. (63) and (65) (blue) and using $\bar{L}_{c}=$ $\bar{L}=100, \bar{R}_{c}=\bar{R}=100$. Dashed lines indicate prediction adding the singular contribution of the surface tension term Eqs. (68a) and (68b) to the previous expression and using the parameters $u_{C z}$ and $\partial_{x x} \zeta_{0}$ or $\partial_{r r} \zeta_{0}$ from the simulations. Dash-dotted lines: full analytical prediction using Eqs. (70a) and (70b). 1D viscosity, $\bar{\eta}=0.5 ; 2 \mathrm{D}$ viscosity, $\bar{\eta}=1$. The results are in normalized units. The critical force in $2 \mathrm{D}$, Eq. (66), provides the maximum value of $\bar{F}_{C z}^{2 D} / \bar{S}$ for the solid blue line and corresponds to $p \approx 3.3 \mathrm{MPa}$.

where the functions $\psi_{1}$ and $\psi_{2}$ defined in Eqs. (C14) and (C15) exhibit the following limits:

$$
\begin{aligned}
& \lim _{z \rightarrow 0} \psi_{1}(z)=\sqrt{2} \ln \frac{1}{z}+C_{1}, \\
& \lim _{z \rightarrow 0} \psi_{2}(z)=C_{2},
\end{aligned}
$$

with $C_{1} \approx 1.645$ and $C_{2} \approx 0.8398$. It follows that when $\zeta_{0} \ll$ $\lambda$ and $\zeta_{0} \ll \lambda \exp \left[-C_{2} /(12 \sqrt{2} \bar{\eta})\right]$, we have

$$
F_{C z}^{1 D} \approx 12 \sqrt{2} \bar{\eta} \frac{L_{c} A}{\lambda} \ln \left(\frac{\lambda}{\zeta_{0}}\right)
$$

This relation indicates that the minimum distance in the contact region decreases exponentially with the applied load in 1D. The prediction Eq. (63) using $L_{c}=L$, which is represented in Fig. 8 by the red solid line, compares well with the numerical results (red circles) when $\zeta_{0}$ is not too small.

In addition, we obtain in $2 \mathrm{D}$ (some details of the derivation are reported in Appendix C2)

$$
\begin{aligned}
\frac{F_{C z}^{2 D}}{\pi R_{c}^{2}}= & {\left[12 \eta D_{e} \frac{A}{\lambda^{3}} \psi\left(\frac{\zeta_{0}}{\lambda}\right) \frac{e^{\frac{\zeta_{0}}{\lambda}}}{1+\frac{\zeta_{0}}{\lambda}}\right.} \\
& \left.+\frac{A}{4 \lambda}\left(\frac{2 \zeta_{0}}{\lambda}+1\right) \frac{e^{-\frac{\zeta_{0}}{\lambda}}}{1+\frac{\zeta_{0}}{\lambda}}\right],
\end{aligned}
$$


where the function $\psi$ obeys

$$
\lim _{z \rightarrow 0} \psi(z)=(1-\ln 2) .
$$

Hence, within this approximation, the LC interface touches the substrate (i.e., $\zeta_{0}=0$ ) for a finite force

$$
F_{c}^{2 D}=\left\{12 \eta D_{e} \frac{A}{\lambda^{3}}[1-\ln (2)]+\frac{A}{4 \lambda}\right\} \pi R_{c}^{2} .
$$

The external force is plotted as a function of $\zeta_{0}$ in Fig. 8 . Equation (65) with $R_{c}=R$ is represented by the blue solid line and has to be compared with the blue squares obtained by direct numerical integration. Once again, this expression agrees with the numerical results for sufficiently large $\zeta_{0}$.

\section{With surface tension}

An inspection of Fig. 8 reveals that the agreement between the predicted force-minimum distance relation and the full numerical solution of thin film equations is accurate only when the forces are not too large. However, as we keep increasing the external load, this prediction (solid lines) fails to reproduce the numerical results. As anticipated previously, the shape of the crystal close to the tip (see inner panel of Fig. 7) is not well described by Eq. (49). Indeed, as $\zeta_{0} \rightarrow 0$, the curvature at the tip diverges, leading to the singular pointy shape reported in Eq. (62). Thus, surface tension effects proportional to the curvature become relevant.

We here resort to a simple matching procedure to account for the consequences of surface tension. First, in the tip region for $x<x_{*}$ or $r<r_{*}$, where $x_{*}$ and $r_{*}$ are the tip width in 1D and 2D respectively, a Taylor expansion of $\zeta$ leads to

$$
\begin{aligned}
& \zeta^{t i p}=\zeta_{0}+\frac{x^{2}}{2} \partial_{x x} \zeta_{0}, \\
& \zeta^{t i p}=\zeta_{0}+\frac{r^{2}}{2} \partial_{r r} \zeta_{0},
\end{aligned}
$$

where $\partial_{r r} \zeta_{0}$ and $\partial_{x x} \zeta_{0}$ are the second derivative of $\zeta$ calculated at $x=0$ or $r=0$.

Using this solution, let us compute the contribution of the tip region to force balance Eqs. (35) and (39). We obtain

$$
\begin{aligned}
& F_{\text {tip }}^{1 D}=2 \frac{A x_{*}}{\lambda}\left(1-\frac{\zeta_{0}}{\lambda}-\partial_{x x} \zeta_{0} \frac{x_{*}^{2}}{6 \lambda}\right)+\eta \frac{6 \pi u_{C z}}{\sqrt{2}\left(\partial_{x x} \zeta_{0}\right)^{3 / 2} \zeta_{0}^{3 / 2}}, \\
& F_{\text {tip }}^{2 D}=\frac{\pi A r_{*}^{2}}{\lambda}\left(1-\frac{\zeta_{0}}{\lambda}-\partial_{r r} \zeta_{0} \frac{r_{*}^{2}}{4 \lambda}\right)+\eta \frac{6 \pi u_{C z}}{\left(\partial_{r r} \zeta_{0}\right)^{2} \zeta_{0}},
\end{aligned}
$$

where we used $\zeta / \lambda \ll 1$ in the tip region. From this expression, it appears that, if $x_{*}$ or $r_{*}$ is not increasing too quickly when the load increases and $\zeta_{0} \rightarrow 0$, the dominant term is the one proportional to the viscosity.

To confirm the validity of this statement, we checked that the increase of the force at small $\zeta_{0}$ is well predicted by adding the singular contribution corresponding to the last term of Eqs. (68a) and (68b) to the previous expressions. The result reported in Fig. 8 agrees well with the deviations at small $\zeta_{0}$. However, this relation is still not fully predictive, since we used $\partial_{x x} \zeta_{0}$ and $\partial_{r r} \zeta_{0}$ obtained from the numerical solution. In order to find an additional relation linking $\zeta_{0}$ and $\partial_{x x} \zeta_{0}$ or $\partial_{r r} \zeta_{0}$, we match the solutions far from and close to the tip in the limit $\zeta \ll \lambda$.

Far from the tip, we assume a small deviation $\delta \zeta$ from the singular solution Eq. (62), leading to $\zeta=\zeta_{\text {sing }}+\delta \zeta$. To find an expression for $\delta \zeta$, we insert the previous relation into the full steady-state differential equation in the presence of curvature terms

$$
\begin{aligned}
& 0=u_{C z}+D_{e} \partial_{x}\left[\zeta\left(\gamma \partial_{x x} \zeta-U^{\prime}(\zeta)\right)\right], \\
& 0=u_{C z}+D_{e} \frac{1}{r} \partial_{r}\left[r \zeta\left(\gamma \partial_{r r} \zeta+\frac{\gamma}{r} \partial_{r} \zeta-U^{\prime}(\zeta)\right)\right],
\end{aligned}
$$

for the $1 \mathrm{D}$ and $2 \mathrm{D}$ respectively. Matching the height and the slope of the tip solution Eq. (67) with the perturbative solution outside the tip region $\zeta=\zeta_{\text {sing }}+\delta \zeta$ at some position $x_{*}$ or $r_{*}$ leads to two equations. These two equations are used to obtain $x_{*}$ or $r_{*}$, and $\partial_{x x} \zeta_{0}$ or $\partial_{r r} \zeta_{0}$, as a function of $\zeta_{0}$. We therefore have a profile with two regions that is completely determined by $\zeta_{0}$. The details of the derivation are quite cumbersome and are therefore reported in Appendix D.

Two important remarks are in order. First, due to the correction $\delta \zeta$, the profile becomes wider when approaching the tip region, in agreement with the shape observed in the full numerical solution in Fig. 7.

As a second remark, the matching analysis shows that $\partial_{x x} \zeta_{0}$ and $\partial_{r r} \zeta_{0}$ tend to a constant for $\zeta_{0} \rightarrow 0$. Using these results in the expression of the force, we obtain asymptotically a powerlaw dependence of the force on $\zeta_{0}$

$$
\begin{aligned}
F_{\text {tip }}^{1 D} & =\frac{12 \pi \eta \gamma^{3 / 2} D_{e} \lambda^{3 / 2}}{C_{1 D}^{3 / 2} \sqrt{2 A} L_{c}^{2}} \frac{1}{\zeta_{0}^{3 / 2}}+\text { nonsingular terms } \\
F_{\text {tip }}^{2 D} & =\frac{24 \pi \eta \gamma^{2} D_{e} \lambda^{2}}{C_{2 D}^{2} A R_{c}^{2}} \frac{1}{\zeta_{0}}+\text { nonsingular terms }
\end{aligned}
$$

where the constants $C_{1 D}=\partial_{\bar{x} \bar{x}} \bar{\zeta}_{0}\left(\bar{\zeta}_{0}=0\right)$ and $C_{2 D}=$ $\partial_{\bar{r} \bar{r}} \bar{\zeta}_{0}\left(\bar{\zeta}_{0}=0\right)$ are the values of the normalized second derivatives at the tip when $\bar{\zeta}_{0} \rightarrow 0$. From simulations, we find $C_{1 D} \approx 0.017$ and $C_{2 D} \approx 0.015$ (see Fig. 11). Note that we used the approximated expression of the dissolution rates $u_{C z}$ given by Eq. (61).

The sum of the contribution without surface tension, Eqs. (63) and (65), with the contribution of the tip, Eqs. (70a) and (70b), are presented in Fig. 8 by the dash-dotted lines. The agreement with the full numerical solution is not quantitative but is satisfactory considering the heuristic character of the matching procedure. Fitting the numerical results with power laws at large forces, we obtain for the wedgelike contact $F_{C z} \sim \zeta_{0}^{-1.3}$ to be compared with the prediction $F_{C z} \sim \zeta_{0}^{-3 / 2}$ from Eq. (70a), while for the conical contact $F_{C z} \sim \zeta_{0}^{-1.1}$ to be compared with $F_{C z} \sim \zeta_{0}^{-1}$ from Eq. (70b).

As a final comment, the critical force for which surface tension becomes relevant is given by Eq. (66) in $2 \mathrm{D}$. In $1 \mathrm{D}$, the comparison of Eqs. (64) and (70a) suggest a critical force

$$
F_{c}^{1 D} \approx 24 \eta D_{e} \frac{A}{2 \lambda^{3}} L_{c},
$$

up to logarithmic corrections. 


\section{DISCUSSION}

\section{A. Summary of results}

In this paper, we have obtained a thin film model describing the evolution of a rigid crystal that is able to grow or dissolve, in the vicinity of a substrate. The model includes hydrodynamics, diffusion, the disjoining pressure effects, and surface tension.

Using this model, we have studied pressure solution against a flat wall in ridgelike (1D) and axisymmetric (2D) contacts. This study has been performed using some simplifying assumptions, including equal density between the liquid and the crystal, the linearization of Gibbs-Thomson relation, and the dilute approximation.

We have also considered two different types of repulsions between the substrate and the crystal. These led to different behaviors.

In the case of a power-law repulsion diverging at contact, the crystal interface flattens under load, and the dissolution rate exhibits a power-law dependence on the load. A change in this power law is found at large loads and viscosities when the forces induced by viscous dissipation surpass those due to disjoining pressure.

In contrast, a finite exponential repulsion produces pointy contacts and a dissolution rate asymptotically independent of the load and the viscosity. For large loads, the sharp pointy shape of the tip is regularized by surface tension, and the force balance is dominated by viscous effects. Touching contact (i.e., $\zeta_{0}=0$ ) is found only in 2D and in the absence of surface tension.

To summarize, we found that for large external loads the dissolution rate $u_{C z}$ and minimum distance $\zeta_{0}$ between the dissolving crystal and the substrate obey scaling laws

$$
\begin{aligned}
& u_{C z} \sim F_{C z}^{\alpha_{u}} L_{c}^{\beta_{u}}, \quad \zeta_{0} \sim F_{C z}^{\alpha_{\zeta}} L_{c}^{\beta_{\zeta}}, \\
& u_{C z} \sim F_{C z}^{\alpha_{u}} R_{c}^{\beta_{u}}, \quad \zeta_{0} \sim F_{C z}^{\alpha_{\zeta}} R_{c}^{\beta_{\zeta}},
\end{aligned}
$$

where $F_{C z}$ is the external load and $L_{c}$ and $R_{c}$ are the contact sizes for the ridge and the axisymmetric contact, respectively. The exponents $\alpha_{u}, \beta_{u}, \alpha_{\zeta}$, and $\beta_{\zeta}$ displayed in Table I are found to depend on dimensionality (ridge or axisymmetric), on viscosity, and on the type of interaction potential (diverging as a power law or finite at contact).

\section{B. Orders of magnitude and model limitations}

Before discussing precise systems, we provide some orders of magnitude describing the energy scale of the interactions. Various experiments and standard textbooks [19] indicate that the order of magnitude of disjoining pressures is typically $U^{\prime} \sim \mathrm{MPa}$ when the distance between the surfaces is $\zeta \sim \mathrm{nm}$. For exponential interactions with decay length $\lambda \sim$ $\mathrm{nm}$ (corresponding, e.g., to the Debye length or to hydration scales), we obtain that $A \sim \lambda U^{\prime} \sim \mathrm{mJ} \mathrm{m}^{-2}$. As a consequence, the dimensionless repulsion strength (see Sec. III A) is $\bar{A}=$ $A / \gamma \sim 10^{-2}$. For power-law interactions, with a typical distance $\lambda \sim$ nm, we have $A \sim U^{\prime} \lambda^{n+1}$. As a consequence, we also find $\bar{A}=A / \gamma \lambda^{n} \sim 10^{-2}$.

We now consider two different crystals: calcite $\mathrm{CaCO}_{3}$ and sodium chlorate $\mathrm{NaClO}_{3}$. For calcite, we use [44,45] solubility $c_{0} \approx 10^{-3} \mathrm{~mol} / 1 \approx 10^{24} / \mathrm{m}^{3}$ (at $25^{\circ} \mathrm{C}$ ), molecular volume $\Omega \approx 100 \AA^{3}$, ionic diffusion constant $D \approx 10^{-5} \mathrm{~cm}^{2} / \mathrm{s}$, watersolution interfacial tension [46] $\gamma \approx 100 \mathrm{~mJ}$, and $T \approx 300 \mathrm{~K}$.

For each variable $y$ in physical units, and the corresponding variable $\bar{y}$ in normalized units, we define the scaling factor $s_{y}$ from the relation $y=s_{y} \bar{y}$. These scaling factors have to be applied to the simulation results to recover physical units. Their precise expressions are given in Appendix B. In the case of calcite, we estimate from Eq. (B3) that

$$
\begin{aligned}
s_{\zeta} & =O(1 \mathrm{~nm}), \quad s_{x}=O(10 \mathrm{~nm}), \\
s_{t} & =O\left(10^{-1} \mathrm{~s}\right), \quad s_{p}=O(\mathrm{MPa}), \\
s_{\eta} & =O\left(10^{2} \mathrm{~Pa} \mathrm{~s}\right) .
\end{aligned}
$$

Considering now $\mathrm{NaClO}_{3}$ with, from Refs. [47,48], $c_{0} \approx 10^{28} / \mathrm{m}^{3}$ (at $\left.25^{\circ} \mathrm{C}\right), \Omega \approx 100 \AA^{3}, D \approx 10^{-5} \mathrm{~cm}^{2} / \mathrm{s}, \gamma \approx$ $10 \mathrm{~mJ}$, and $T \approx 300 \mathrm{~K}$, and using the same assumption on the interaction range and strength, we have $\bar{A}=10^{-1}$ and

$$
\begin{aligned}
& s_{\zeta}=O(1 \mathrm{~nm}), \quad s_{x}=O(1 \text { to } 10 \mathrm{~nm}), \\
& s_{t}=O\left(10^{-6} \mathrm{~s}\right), \quad s_{P}=O(\mathrm{M} \mathrm{Pa}) \\
& s_{\eta}=O\left(10^{-2} \mathrm{~Pa} \mathrm{~s}\right)
\end{aligned}
$$

As an illustrative example for the use of these scaling

\begin{tabular}{|c|c|c|c|c|c|c|}
\hline \multirow{2}{*}{$\frac{\text { Repulsion }}{\text { Regime }}$} & \multicolumn{2}{|c|}{ Power law } & \multicolumn{4}{|c|}{ Exponential } \\
\hline & $\begin{array}{l}\text { Hydrodyn. } \\
\bar{\eta} \geqslant 1\end{array}$ & $\begin{array}{c}\text { Diffusion. } \\
\bar{\eta} \ll 1\end{array}$ & $\begin{array}{c}\text { 1D no surf. tens. } \\
F_{C z}^{1 D} \ll F_{c}^{1 D}\end{array}$ & $\begin{array}{l}\text { 1D surf. tens. } \\
F_{C z}^{1 D} \gg F_{c}^{1 D}\end{array}$ & $\begin{array}{l}\text { 2D no surf. tens. } \\
\qquad F_{C z}^{2 D}<F_{c}^{2 D}\end{array}$ & $\begin{array}{l}\text { 2D surf. tens. } \\
F_{C z}^{2 D} \gg F_{c}^{2 D}\end{array}$ \\
\hline$\alpha_{u}$ & $\frac{n}{n+3}$ & $\frac{n}{n+1}$ & 0 (constant) & 0 (constant) & 0 (constant) & 0 (constant) \\
\hline$\beta_{u}$ & $-\frac{4 n+6}{n+3}$ & $-\frac{4 n+2}{n+1}$ & -2 & -2 & -2 & -2 \\
\hline$\alpha_{\zeta}$ & $-\frac{1}{n+3}$ & $\frac{-1}{n+1}$ & Exponential & $-2 / 3$ & $\zeta_{0} \rightarrow 0$ as $F_{C z}^{2 D} \rightarrow F_{c}^{2 D}$ & -1 \\
\hline$\beta_{\zeta}$ & $\frac{2}{n+3}$ & $\frac{2}{n+1}$ & Exponential & $-4 / 3$ & & -2 \\
\hline
\end{tabular}
factors, simulations were performed in a box of normalized width 100 with an initial distance equal to 1 between the dissolving crystal and the substrate. For both cases of calcite and sodium chlorate, this corresponds to thicknesses of the

TABLE I. Summary of the asymptotic regimes at large loads following the notation of Eq. (71). For exponential potentials in the absence of surface tension effects, the dependence of $u_{C z}$ and $\zeta_{0}$ on the load and system size is not a power law. In $1 \mathrm{D}$, the dependence is logarithmic, Eq. (64), and in 2D $\zeta_{0}$ vanishes (i.e., the crystal touches the substrate) for a finite force $F_{c}^{2 D}$, Eq. (66). 
order of the nanometer. In addition, contact widths are $\sim 1 \mu \mathrm{m}$ for calcite, and $\sim 100 \mathrm{~nm}$ to $1 \mu \mathrm{m}$ for sodium chlorate.

Some remarks are in order. First, the order of magnitude of the relevant pressures does not depend much on the system. In contrast, the order of magnitude of the time scale and of the relevant viscosities depend strongly on the solubility $c_{0}$, which can vary by many orders of magnitude from one material to another.

As discussed previously for dissolution with singular (power-law) repulsions, one could discriminate between diffusive and hydrodynamic regimes. The simulation results show that the high-viscosity regime (hydrodynamic regime) is expected for $\bar{\eta} \geqslant 1$ (top panel of Fig. 5) for $F_{C z} / S \sim$ $10^{2} \mathrm{MPa}$ to $10 \mathrm{GPa}$ with $S=\pi R^{2}$, and micrometric crystals $(\bar{R}=100 \leftrightarrow R=1 \mu \mathrm{m})$. For calcite, this would be expected for $\eta \sim 10^{2} \mathrm{~Pa} \mathrm{~s}$, which is much larger of the value for water $(\approx \mathrm{mPa}$ s). As a consequence, for this system the observation of such regime should be difficult in natural environments. However, for highly soluble salts such as $\mathrm{NaClO}_{3}$, we would need $\eta \sim 10 \mathrm{mPa}$, much closer to the value of water. Therefore, the hydrodynamic dissolution regime should be easier to observe in this type of systems.

However, physical parameters such as viscosity and diffusion can also depend on pressure, temperature, and $\mathrm{pH}$ or be affected by phenomena inherent to confinement. For example, large pressures are know to lead to variation of the viscosity [49] while nano confinement when double layer is present on the surfaces could promote higher effective viscosities (electroviscosity) [50].

One should keep in mind that there are limits in the application of our continuum model. For instance, when $\zeta_{0}$ reaches the molecular scale, the continuum approach will break down and one should resort to different models based on molecular methods. An interesting step in this direction was recently proposed in the literature using kinetic Monte Carlo simulations [51]. Atomistic simulations may also allow one to tackle discontinuities of the surface profile such as atomic steps, which where shown to be relevant for pressure solution experiments [52].

Moreover, one of the approximations used in our study of pressure solution is the linearization of the Gibbs-Thomson relation. The full nonlinear expression of the Gibbs-Thomson relation must be kept when $U^{\prime}(\zeta) \ll k_{B} T / \Omega$. At room temperature, $k_{B} T / \Omega \sim 1 \mathrm{MPa}$ for molecular crystals and $k_{B} T / \Omega \sim$ $1 \mathrm{GPa}$ for atomic crystals. As discussed at the beginning of this section, we may assume maximum disjoining pressures $U^{\prime}$ of the order of the $\mathrm{MPa}$, and the assumption $U^{\prime}(\zeta) \ll k_{B} T / \Omega$, although not systematically valid, should apply in many cases. As discussed in Appendix E, our analysis can be extended to the case where the full nonlinearity of the Gibbs-Thomson relation is kept. This leads to similar results as those discussed above in the presence of an exponential potential. The only important difference appears for power-law interactions: The functional form of the dissolution rate and minimum distance with the force are not power law anymore. Instead, they exhibit an essential singularity, as discussed in Appendix E.

Another limitation of our model is the absence of elastic or plastic displacements in the solid. However, our results show that even in the absence of elasticity or plasticity, significant shape changes can be observed in contact zones due to dissolution or growth kinetics in the presence of disjoining pressure effects. Hence, elasticity or plasticity are not the only pathways toward flat contact shapes in pressure solution, and dissolution alone is a sufficient mechanism. Beyond displacements, elasticity also gives rise to an additional contribution to the chemical potential [53] $\Omega \sigma^{2} / 2 E$, where $E$ is the Young modulus. For this contribution to be dominant as compared to that coming from disjoining pressure $\Omega U^{\prime}$, one should have stresses larger than $\left(2 E U^{\prime}\right)^{1 / 2}$. Taking $U^{\prime} \sim \mathrm{MPa}$, and $E \sim 10 \mathrm{GPa}$, we obtain that stresses should typically exceed $10^{2} \mathrm{MPa}$ for elastic effects to be relevant in the chemical potential. In addition, pointy morphologies such as those obtained in our model for finite repulsions should lead to a concentration of stresses, which could result in significant elastic or plastic effects. Further studies in this direction are needed.

Finally, one major assumption of our study is the constant size of the contact region. While specific needlelike crystal shapes may indeed present a constant contact area during dissolution, it is clear that more general shapes, e.g., conical or spherical crystals, would exhibit a growing contact area as dissolution proceeds. In addition, redeposition of material ouside the contact could also change the contact area during pressure solution. Our description could still hold if the change in the contact area was slower than the relaxation of the crystal profile within the contact. Such a separation of time scales, where a steady state is reached within the contact as if the contact size was constant at all times, will be denoted as the quasistatic approximation.

In the following, we discuss the validity of this approximation. Effects such as redeposition, growth, or dissolution outside the contact are assumed to be smaller than the dissolution in the contact region. From dimensional analysis of Eq. (40), neglecting the contribution of surface tension, the relaxation time $t_{\text {relax }}$ toward a steady-state profile $\zeta_{s}(r)$ with a contact of size $R_{c}$ is $t_{\text {relax }} \sim R_{c}^{2} /\left[D_{e} \tilde{U}^{\prime}\left(\zeta_{s}\right)\right]$. In addition from force balance, Eq. (39), we have $F \sim R_{c}^{2} U^{\prime}\left(\zeta_{s}\right)$. Since $U^{\prime}\left(\zeta_{s}\right) \sim \tilde{U}^{\prime}\left(\zeta_{s}\right)$, we find $t_{\text {relax }} \sim R_{c}^{4} /\left(D_{e} F\right)$. Assuming a small contact angle $\theta_{\text {ext }}$ at the edge of the contact, dissolution induces a growth velocity for the contact radius $d R_{c} / d t=$ $u_{C z} / \theta_{\text {ext }}$. We must therefore require that the relaxation time is smaller than the time associated with the growth of the contact radius: $t_{\text {relax }} \ll R_{c} /\left(d R_{c} / d t\right)$, leading to $R_{c}^{4} /\left(D_{e} F\right) \ll$ $R_{c} \theta_{\text {ext }} / u_{C z}$. For example, in the case of a power-law potential in the diffusion-dominated regime, $u_{C z}$ is given by Eq. (56b), and this condition leads to $F \gg A /\left(\theta_{\mathrm{ext}}^{n+1} R_{c}^{n-1}\right)$. Using the relation stated above in this subsection, $A \sim U^{\prime} \lambda^{n+1}$, and the force balance $F \sim R_{c}^{2} U^{\prime}$, we finally obtain a simple condition $\lambda / R_{c} \ll \theta_{\text {ext }}$. Since we assumed $\lambda / R_{c} \sim 10^{-5}$ above (with $\lambda \sim$ nm and $R_{c} \approx 100 \mu \mathrm{m}$ ), this result suggests that for contact angles not too small $\theta_{\text {ext }} \gg 10^{-5}$, the quasistatic approximation should be valid.

Within this approximation, the dissolution rate will depend on the shape of the dissolving solid. For example, for a cone of half angle $\theta_{\text {cone, }}$, assuming no redeposition outside the contact region, the radius of the contact area obeys $d R_{c} / d t=u_{C z} \tan \theta_{\text {cone }}$. Similarly, for a sphere of radius $R_{0}$, we have $d R_{c} / d t=u_{C z}\left(R_{0}^{2} / R_{c}^{2}-1\right)^{1 / 2}$. Since $u_{C z} \sim R_{c}^{\beta_{u}}$ from 
Eq. (71b), we find that $R_{c} \sim t^{1 /\left(1-\beta_{u}\right)}$ and $u_{C z} \sim t^{\beta_{u} /\left(1-\beta_{u}\right)}$ at constant force in the conical case, and $R_{c} \sim t^{1 /\left(2-\beta_{u}\right)}$ and $u_{C z} \sim$ $t^{\beta_{u} /\left(2-\beta_{u}\right)}$ at constant force in the spherical case when $R_{c} \ll$ $R_{0}$. Choosing again the example of power-law repulsion in the diffusion limited regime where $\beta_{u}=-(4 n+2) /(n+1)$, we find $u_{C z} \sim t^{-(4 n+2) /(5 n+3)}$ and $u_{C z} \sim t^{-(2 n+1) /(3 n+2)}$ for the conical and spherical cases respectively.

\section{Comparison with existing models and experiments}

Since it relates deformation strains, contact size, and stress on single-contact dissolution (eventually connecting it to the overall grain compaction problem) in an axisymmetric geometry, Weyl's model [12] is a first natural candidate for comparison to our model. Weyl predicts that $u_{C z}=8 D \lambda b F_{C z} / R_{c}^{2}$, where $D$ is the diffusion constant, $\lambda$ is the film thickness, $b$ is a linear stress coefficient linking local solute concentration with the applied stress, and $R_{c}$ is the contact size.

Other models consider the phenomena at the scale of the grain rather than the contact region [13,14,16-18]. Rutter [15] summarizes most of the previously cited models (for diffusion-controlled kinetics) and also treats the global problem at the thin film contact area, as done by Weyl. In cylindrical symmetry and for small external stresses, Rutter [15] predicts $u_{C z}=32 C_{0} D w V F_{C z} /\left(\mathcal{R}_{g} T \rho_{C} d^{3}\right)$, while for high external stresses $(>100 \mathrm{MPa})$ Rutter finds $u_{C z}=$ $40 c_{0} D w \exp \left[F_{C z} V /\left(2.3 \mathcal{R}_{g} T\right)\right] /\left(d^{3} \rho_{C}\right)$, where $c_{0}$ is the concentration at the interface, $\rho_{C}$ is the crystal density, $D$ is the diffusion at the grain boundary, $w$ is an effective width, $\mathcal{R}_{g}$ is the gas constant, and $d$ is the grain size (proportional to the contact size).

The relations predicted by Weyl and Rutter are in general not in agreement with our predictions both for power-law repulsion and finite exponential repulsion, Eqs. (55b), (56b), and (61b).

Globally, the absence of description of microscopic physical ingredients such as viscosity, interaction potential, and surface tension in these models lead to a very different and nonspecific behavior.

Previous modeling attempts have also addressed the regime of slow interface kinetics [17]. They suggest that the dissolution rate could then be independent of the contact area. The investigation of this limit is an interesting perspective for further development of our model.

A number of experimental observations have suggested power-law relations between strain rates (crystal velocity) and applied stress and or grain size [54-56]. This is compatible with the results we obtained for the singular repulsive power-law potential in Eqs. (55a), (55b), (56a), and (56b) and Fig. 5. However, Croizé et al. [57] underline that though there exists a positive correlation between the strain rate and the applied stress, this dependence is weak. With the support of both original measurements on calcite pressure solution and data from the literature, they claim that other effects such as the grain size are likely to be dominant. These observations are consistent with the scenario predicted for exponential interaction in Eq. (61) and Fig. 6.

Using the pressure range 1 to $10^{3} \mathrm{MPa}$, which is the one usually considered in pressure solution experiments, the velocities (dissolution rates) obtained by our simulations are $10^{-3}$ to $10^{-1} \mathrm{~nm} \mathrm{~s}^{-1}$ for calcite and $10^{-1}$ to $10 \mu \mathrm{m} \mathrm{s}^{-1}$ for sodium chlorate. The observable usually reported in pressure solution experiments is the strain rate. Experimental values of the strain rates for calcite [54,57] vary between $10^{-9}$ and $10^{-4} \mathrm{~s}^{-1}$. Using $\dot{\epsilon}=u_{C z} / R_{c}$ as the definition of the strain rate [57], we obtain values between $10^{-6}$ and $10^{-4} \mathrm{~s}^{-1}$, compatible with the experimental ones. For $\mathrm{NaClO}_{3}$, because of the faster time scales due to the much higher solubility, the dissolution rate and as a consequence the strain rate increases of a factor of about $10^{5}$. This is in disagreement with the literature [56], where similar orders of magnitude as those of calcite are found. Such discrepancy could be caused by the fact that in our system exhibits an undersaturated concentration bath at the boundaries of the contact. In multicontact systems where the liquid reservoir per contact is finite, the global supersaturation of the bath should increase due to the release of crystal molecules in the liquid. This should lead to a decrease of the dissolution rates. The study of such interactions between different contacts is therefore an important perspective for our modeling approach to address systems with multiple contacts.

As far as the morphology of the contact is concerned, some experiments on quartz grains aggregates [58] showed that in addition to relative smooth interfaces, irregular ridge and plateau structures can develop at the grain contacts after undergoing pressure solution. The appearance of pointlike and ridgelike singularities for exponential repulsions in our model could be a first step toward the understanding of these morphologies.

In general, further experimental investigation involving observations at the scale of one microscopic contact would be useful to test our model predictions.

\section{CONCLUSIONS}

In conclusion, we have presented a thin film model for the dynamics of lubricated contacts during dissolution and growth under load, accounting for surface tension, interactions, diffusion, and hydrodynamics. This model describes the coupled evolution of the space-dependent pressure field $p$ in the liquid and the film thickness $\zeta$ via Eqs. (27) and (30). An additional constraint originating in global force balance Eqs. (32) and (33) determines the crystal velocity $\mathbf{u}_{C}$.

Using this model, we have discussed the dynamics of pressure solution for single contacts of fixed or slowly varying size and with symmetric geometries, using some simplifying assumptions. We find that the dissolution rate and contact morphology exhibit distinctive behaviors depending on the finiteness of the repulsion at contact. Furthermore, we find that crystal-substrate touching contact is never reached in steady state for any load when viscosity and surface tension are taken into account.

Much yet remains to be done to explore the different regimes emerging for our thin-film model. However, this model paves the way for a systematic and physically consistent analysis of the influence of different microscopic ingredients on pressure solution and growth in confined environments.

\section{ACKNOWLEDGMENTS}

The authors wish to thank Alois Meckenstock for useful comments on the manuscript. This project has received 
funding from the European Union's Horizon 2020 research and innovation program under Grant Agreement No. 642976.

\section{APPENDIX A: IDENTITIES RESULTING FROM TRANSLATIONAL INVARIANCE OF THE FREE ENERGY}

Here we derive some integral identities that are used in the main text. These identities express the fact that the total force resulting from a translational-invariant energy must vanish.

Consider a generic free energy functional $\mathcal{F}_{\mathcal{D}}$ acting over a domain $\mathcal{D}$ in $d$ dimensions and with boundary $\partial \mathcal{D}$ in $(d-1)$ dimensions. Let us assume that its variation can be written as a surface integral

$$
\delta \mathcal{F}=\int_{\partial \mathcal{D}} d S(\delta \mathbf{r} \cdot \hat{\mathbf{n}}) \frac{\delta \mathcal{F}_{\mathcal{D}}}{\delta \mathbf{r}},
$$

where $\delta \mathbf{r}$ is a $d$-dimensional infinitesimal variation of the domain boundary.

Assume now that $\mathcal{F}_{\mathcal{D}}$ is invariant under translations. Then, $\delta \mathcal{F}$ must vanish under infinitesimal translations, i.e., when $\delta \mathbf{r}=d \mathbf{r}$ is an arbitrary constant (independent on space coordinates). As a consequence,

$$
0=d \mathbf{r} \cdot \int_{\partial \mathcal{D}} d S \hat{\mathbf{n}} \frac{\delta \mathcal{F}_{\mathcal{D}}}{\delta \mathbf{r}} .
$$

Since this is true for any $d \mathbf{r}$, we find that the force acting on the domain surface vanishes:

$$
0=\int_{\partial \mathcal{D}} d S \hat{\mathbf{n}} \frac{\delta \mathcal{F}_{\mathcal{D}}}{\delta \mathbf{r}} .
$$

This relation is valid for arbitrary shapes of the domain $\mathcal{D}$.

In particular, consider the surface energy

$$
\mathcal{F}_{\mathcal{S}}=\int_{\partial \mathcal{D}} d S \gamma(\hat{\mathbf{n}})
$$

whose variation is given by

$$
\int_{\partial \mathcal{D}} d S \hat{\mathbf{n}}(\kappa: \tilde{\gamma})=0,
$$

where $\gamma$ is a general surface tension (function of the orientation), $\tilde{\gamma}$ is the stiffness tensor, and $\kappa$ is the curvature tensor. In the special case where the surface tension is isotropic, i.e., $\gamma$ does not depend on $\hat{\mathbf{n}}$, we obtain a known equality: The integral of the mean curvature times the normal vector of an arbitrary (sufficiently regular) surface vanishes [59]

$$
\int_{\partial \mathcal{D}} d S \hat{\mathbf{n}} H=0
$$

where $H$ is the mean curvature.

Finally, another useful relation is obtained when choosing an energy proportional to the volume of the domain $\mathcal{D}$ :

$$
\int_{\partial \mathcal{D}} d S \hat{\mathbf{n}}=0 .
$$

We find that the integral of the normal vector vanishes on any closed regular surface.

\section{APPENDIX B: RESCALING AND UNITS}

Let us recall the type of substrate-crystal interactions considered, Eqs. (41) and (42):

$$
\begin{aligned}
& U(\zeta)=\frac{A}{\zeta^{n}} \quad \text { Singular at contact, } \\
& U(\zeta)=A e^{-\frac{\zeta}{\lambda}} \quad \text { Finite at contact. }
\end{aligned}
$$

For simplicity, we only show the scaled equations in 1D. In the case of the power-law repulsion, Eq. (41) with $n=3$, we have

$$
\begin{aligned}
& \partial_{\bar{t}} \bar{\zeta}=-\partial_{\bar{x}}\left[\bar{\zeta} \partial_{\bar{x}}\left(\partial_{\bar{x} \bar{x}} \bar{\zeta}+\frac{1}{\bar{\zeta}^{4}}\right)\right]-\bar{u}_{C z}, \\
& \bar{u}_{C z} \int_{0}^{\bar{L}} d \bar{x} \int_{\bar{x}}^{\bar{L}} d \bar{x}^{\prime} \frac{24 \bar{\eta} \bar{x}^{\prime}}{\bar{\zeta}^{3}}=\bar{F}_{C z}+2 \int_{0}^{\bar{L}} d \bar{x} \frac{1}{\bar{\zeta}^{4}},
\end{aligned}
$$

where $\bar{u}_{C z}, \bar{\eta}$, and $\bar{F}$ are the rescaled velocity, viscosity, and external force, respectively. For the exponential repulsion, Eq. (42), we have

$$
\begin{aligned}
& \partial_{\bar{t}} \bar{\zeta}=-\partial_{\bar{x}}\left[\bar{\zeta} \partial_{\bar{x}}\left(\partial_{\bar{x} \bar{x}} \bar{\zeta}+e^{-\bar{\zeta}}\right)\right]-\bar{u}_{C z} \\
& \bar{u}_{C z} \int_{0}^{\bar{L}} d \bar{x} \int_{\bar{x}}^{\bar{L}} d \bar{x}^{\prime} \frac{24 \bar{\eta} \bar{x}^{\prime}}{\bar{\zeta}^{3}}=\bar{F}_{C z}+2 \int_{0}^{\bar{L}} d \bar{x} e^{-\bar{\zeta}} .
\end{aligned}
$$

If $y$ is an arbitrary variable and $\bar{y}$ is its normalized counterpart used in simulations, we define $s_{y}$ the scaling factor that has to be applied to recover the natural variables from the normalized simulation variables: $y=s_{y} \bar{y}$. Defining $\bar{A}$ as a nondimensional quantity equal to $A / \gamma$ for the exponential repulsion, and equal to $A /\left(\gamma \lambda^{3}\right)$ for the power-law repulsion, the scaling factors are for both Eqs. (B1) and (B2)

$$
\begin{aligned}
s_{\zeta} & =\lambda, \\
s_{x} & =\lambda\left(\frac{1}{\bar{A}}\right)^{1 / 2}, \\
s_{t} & =\lambda^{3} \frac{k_{B} T}{D \Omega^{2} c_{0} \gamma \bar{A}^{2}}, \\
s_{F}^{1 D} & =\gamma \bar{A}^{1 / 2}, \\
s_{F}^{2 D} & =\gamma \lambda, \\
s_{p} & =\frac{\gamma \bar{A}}{\lambda} \\
s_{\eta} & =\lambda^{2} \frac{k_{B} T}{D \Omega^{2} c_{0}}, \\
s_{u_{C z}} & =\frac{D \Omega^{2} c_{0} \gamma \bar{A}^{2}}{k_{B} T \lambda^{2}} .
\end{aligned}
$$

The superscripts $1 D$ and $2 D$ explicitly indicate those scalings which differ in the ridge and axisymmetric system. Also note that $s_{p}=s_{F}^{1 D} / s_{x}$ in $1 \mathrm{D}$, while $s_{p}=s_{F}^{2 D} / s_{x}^{2}$ in $2 \mathrm{D}$. 


\section{APPENDIX C: STEADY STATE IN THE ABSENCE OF SURFACE TENSION}

We here illustrate how to derive some relations of Secs. IV A and IV B 1 for the axisymmetric system (2D). An analogous procedure can be followed in $1 \mathrm{D}$. In $2 \mathrm{D}$, a single integration of Eq. (46) leads to

$$
\frac{r}{2} u_{C z}=D_{e} \zeta \partial_{r} \zeta U^{\prime \prime}(\zeta)
$$

Using the previous relation to express the differential $r d r$ as a function of $d \zeta$ and considering only the contribution of the contact area, we can rewrite Eq. (39) in a more convenient form:

$$
\begin{aligned}
F_{C z}= & 2 \pi \int_{0}^{R_{c}} r 12 \eta D_{e} d r \int_{\zeta(r)}^{\zeta\left(R_{c}\right)} d \zeta \frac{U^{\prime \prime}(\zeta)}{\zeta^{2}} \\
& -2 \pi \int_{0}^{R_{c}} d r r U^{\prime}(\zeta(r)) .
\end{aligned}
$$

\section{Singular power-law repulsion}

Using Eq. (51) together with Eq. (41) in Eq. (C2), we find

$$
\begin{aligned}
F_{C z}^{2 D}= & 12 \eta C_{1}\left(r_{m}, R_{c}\right)\left(R_{c}^{2} u_{C z}\right)^{\frac{n+3}{n}} \\
& +C_{2}\left(r_{m}, R_{c}\right)\left(R_{c}^{2} u_{C z}\right)^{\frac{n+1}{n}},
\end{aligned}
$$

where

$$
\begin{aligned}
C_{1}= & D_{e} \frac{n(n+1) \pi A}{n+3}\left\{\frac{-R_{c}^{2}}{\zeta^{n+3}\left(R_{c}\right)}+\frac{n r_{m}^{2}}{(2 n+3) \zeta_{0}^{n+3}}\right. \\
& \left.\times\left[\left(\frac{R_{c}^{2}}{r_{m}^{2}}-1\right)^{\frac{n+3}{n}}+1\right]\left(\frac{r_{m}^{2} / R_{c}^{2}}{4 D_{e}(n+1) A}\right)^{\frac{n+3}{n}}\right\}, \\
C_{2}= & \frac{\pi n^{2} A r_{m}^{2}}{(2 n+1)}\left[\left(\frac{R_{c}^{2}}{r_{m}^{2}}-1\right)^{\frac{2 n+1}{n}}+1\right]\left(\frac{r_{m}^{2} / R_{c}^{2}}{4 D_{e}(n+1) A}\right)^{\frac{n+1}{n}} .
\end{aligned}
$$

For large external loads, we have $r_{m} \sim R_{c}$ and $\zeta\left(r=R_{c}\right) \gg$ $\zeta_{0}$, leading to Eq. (54).

The constants used in the main text in Eqs. (55a), (55b), (56a), and (56b) were obtained considering that one of the two terms in Eq. (C3) dominates in the force balance depending on the value of the viscosity. Their expressions are

$$
\begin{aligned}
C_{h}^{1 D} & =\frac{2\left[D_{e} A(n+1)\right]^{\frac{3}{n+3}}}{\left[\frac{24 n \sqrt{\pi}}{n+3} \phi\left(\frac{n+3}{n}\right)\right]^{\frac{n}{n+3}}}, \\
C_{h}^{2 D} & =\frac{4\left[D_{e} A(n+1)\right]^{\frac{3}{n+3}}}{\left[\frac{12 \pi n^{2}}{(2 n+3)(n+3)}\right]^{\frac{n}{n+3}}}, \\
C_{d}^{1 D} & =\frac{2 D_{e} A^{\frac{1}{n+1}}(n+1)}{\left[2 n \sqrt{\pi} \phi\left(\frac{n+1}{n}\right)\right]^{\frac{n}{n+1}}}, \\
C_{d}^{2 D} & =\frac{4 D_{e} A^{\frac{1}{n+1}}(n+1)}{\left(\frac{\pi n^{2}}{2 n+1}\right)^{\frac{n}{n+1}}} .
\end{aligned}
$$

As discussed in the main text, the force can also be written as a function of the distance $\zeta_{0}$ between the substrate and the
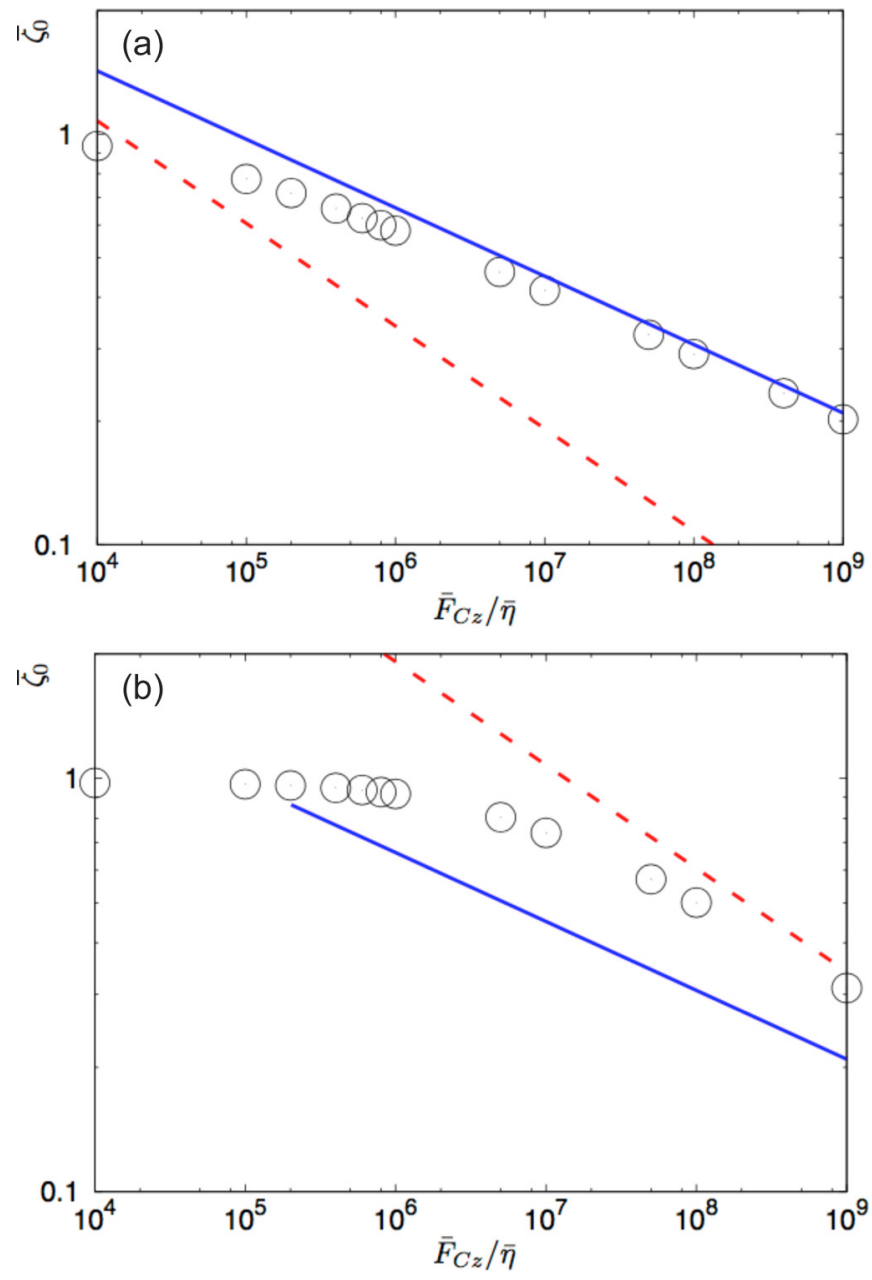

FIG. 9. Minimum film thickness $\zeta_{0}$ as a function of the applied load for (singular) power-law repulsion. Geometry: axisymmetric contact. Lines represent analytical predictions extracted from Eq. (C8) with $\bar{R}_{c}=\bar{R}=100$; circles indicate simulation results. Solid blue line, hydrodynamic regime; dashed red line, diffusion regime. (a) $\bar{\eta}=1$; (b) $\bar{\eta}=10^{-3}$. The results are given in normalized units.

crystal surface at the center of the contact:

$$
\begin{aligned}
\frac{F_{C z}^{2 D}}{\pi R_{c}^{2}}= & 12 \eta D_{e} \frac{n^{2}(n+1) A}{(2 n+3)(n+3)}\left(\frac{1}{\zeta_{0}}\right)^{n+3} \\
& +\frac{n^{2} A}{(2 n+1)}\left(\frac{1}{\zeta_{0}}\right)^{n+1}
\end{aligned}
$$

leading to the asymptotic scaling reported in Sec. V A. These results are confirmed by the numerical solution as showed in Fig. 9.

Finally, as showed in Fig. 10, we have explored the transition between the diffusion and hydrodynamic scaling laws. This was done using an intermediate viscosity, $\bar{\eta}=0.1$, and looking at the dissolution rates in a 2 D contact of size $\bar{R}_{c} \approx \bar{R}=100$. 


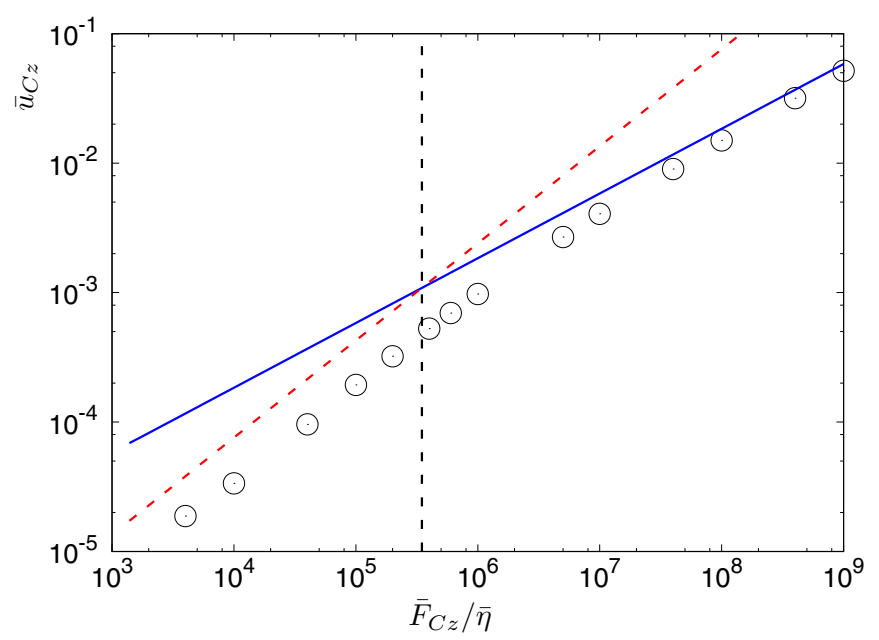

FIG. 10. Dissolution rate as a function of the applied load for power-law (singular) repulsion. Geometry: axisymmetric contact. The viscosity is $\bar{\eta}=10^{-1}$. Lines represent analytical predictions with $\bar{R}_{c}=\bar{R}=100$, circles indicate simulation results. Solid blue line, hydrodynamic regime, Eq. (55b); dashed red line, diffusion regime, Eq. (56b). The black dashed line represents the expected threshold between the two regimes according to Eq. (58). The results are shown in normalized units.

The constants appearing in Eqs. (57) and (58) are

$$
\begin{gathered}
B_{1 D}=\left\{\frac{24 D_{e}(n+1) \phi\left(\frac{n+3}{n}\right)}{(n+3)\left[2 \phi\left(\frac{n+1}{n}\right)\right]^{\frac{n+3}{n+1}}}\right\}^{\frac{n+1}{2}} \frac{1}{n A \sqrt{\pi}}, \\
B_{2 D}=\left[\frac{12 D_{e}(n+1)(2 n+1)^{\frac{n+3}{n+1}}}{(2 n+3)(n+3)}\right]^{\frac{n+1}{4}} \frac{1}{n \sqrt{A \pi}} .
\end{gathered}
$$

From Eq. (58), with $\bar{R}_{*}=100, \bar{\eta}=0.1, n=3$ (since in simulations units $D_{e}=1$ and $A=1 / 3, B_{2 D} \approx 5.4$ ), we expect the diffusion limited regime approximately for $\bar{F}_{C z}<3.5 \times$ $10^{4}$ and the hydrodynamic regime otherwise. The threshold indicated in the figure by the dashed vertical line is compatible with the observed trend.

\section{Finite exponential repulsion}

In the case of a finite exponential repulsion, manipulations similar to those presented in the previous section lead to the following form of the force balance relation:

$$
F_{C z}^{2 D}=48 \eta \frac{\pi D_{e}^{2} A^{2}}{\lambda^{3} u_{C z}} \psi\left(\frac{\zeta_{0}}{\lambda}\right)+\frac{\pi D_{e} A^{2}}{\lambda}\left(\frac{2 \zeta_{0}}{\lambda}+1\right) \frac{e^{-\frac{2 \zeta_{0}}{\lambda}}}{u_{C z}},
$$

with

$$
\psi\left(z_{0}\right)=\lambda \int_{z_{0}}^{\infty} d z e^{-z}\left[e^{-z}+z E i(-z)\right],
$$

where $E_{i}$ is the exponential integral defined as

$$
E i(x)=-\int_{-x}^{\infty} \frac{e^{-s}}{s} d s .
$$

Inserting the expression of $u_{C z}$ from Eq. (61b) into Eq. (C11), we obtain Eq. (65).

\section{1D case}

In $1 \mathrm{D}$, the derivations are similar to the $2 \mathrm{D}$ case. We obtain Eq. (63), where the two functions $\tilde{\psi}_{1}$ and $\tilde{\psi}_{2}$ are defined as

$$
\begin{aligned}
& \tilde{\psi}_{1}\left(z_{0}\right)=\int_{z_{0}}^{\infty} \frac{e^{-z}\left[e^{-z}+z E_{i}(-z)\right]}{\left[\left(1+z_{0}\right) e^{-z_{0}}-(1+z) e^{-z}\right]^{\frac{1}{2}}} d z \\
& \tilde{\psi}_{2}\left(z_{0}\right)=\int_{z_{0}}^{\infty} \frac{z e^{-2 z}}{\left[\left(1+z_{0}\right) e^{-z_{0}}-(1+z) e^{-z}\right]^{\frac{1}{2}}} d z
\end{aligned}
$$

\section{APPENDIX D: SURFACE TENSION CONTRIBUTION IN FINITE REPULSION}

We here report a derivation of the relation between the second derivative of the interface $\partial_{x x} \zeta_{0}$ or $\partial_{r r} \zeta_{0}$ and the minimum film width $\zeta_{0}$. This relation is obtained through a procedure where we match the two approximate solutions at the tip $\zeta_{\text {tip }}$ in Eq. (67), and far from the tip $\zeta_{\text {sing }}$ in Eq. (62).

\section{1D case}

In $1 \mathrm{D}$ we proceed as follows. Integrating two times Eq. (69a), we have

$$
\begin{aligned}
0= & \frac{x^{2}}{2 D_{e}} u_{C z}-\frac{A}{2 \lambda}\left(\zeta^{2}-\zeta_{0}^{2}\right) \\
& +\gamma\left[\zeta \partial_{x x} \zeta-\zeta_{0} \partial_{x x} \zeta_{0}-\frac{1}{2}\left(\partial_{x} \zeta\right)^{2}\right],
\end{aligned}
$$

where we used the parity condition $\partial_{x} \zeta_{0}=0$ and the expansion of $\tilde{U}$ [given for the exponential repulsion by Eq. (59)], up to second order in $\zeta: \tilde{U} \approx A\left[-1+\zeta^{2} /\left(2 \lambda^{2}\right)\right]$.

Adding a perturbation $\delta \zeta$ to $\zeta_{\text {sing }}=\omega|x|$ given by Eq. (62a), we have

$$
\zeta_{\text {far }}=\zeta_{\text {sing }}+\delta \zeta
$$

with

$$
\omega=\left(\frac{u_{C z}}{D_{e} A}\right)^{\frac{1}{2}} \lambda .
$$

We then insert this relation in Eq. (D1) to determine $\delta \zeta$ far from the tip. Neglecting the terms of smaller than $\delta \zeta$ for large $x$, we find

$$
\delta \zeta=\gamma \frac{-\frac{1}{2} \omega^{2}-\zeta_{0} \partial_{x x} \zeta_{0}}{\frac{A}{\lambda^{2}} \omega x} .
$$

We define $x_{*}$ as the value of $x$ at which we match the solutions $\zeta_{\text {sing }}$ and $\zeta_{\text {tip }}$. We obtain two independent relations. The first one accounts for the matching of the surface profiles at $x=x_{*}$, leading to $\omega x_{*}+\delta \zeta\left(x_{*}\right)=\zeta_{0}+\partial_{x x} \zeta_{0} x_{*}^{2} / 2$. The second relation comes from the matching of the slopes $\omega+$ $\left.\partial_{x} \delta \zeta(x)\right|_{x_{*}}=\partial_{x x} \zeta_{0} x_{*}$. Combining the two relations, we obtain 
the following system of equations:

$$
\begin{aligned}
\frac{3}{2} \partial_{x x} \zeta_{0} x_{*}^{2}-2 \omega x_{*}+\zeta_{0} & =0 \\
\omega x_{*}-\gamma\left(\frac{\omega^{2}}{2} \zeta_{0} \partial_{x x} \zeta_{0}\right) \frac{\lambda^{2}}{A \omega x_{*}} & =\zeta_{0}+\frac{\partial_{x x} \zeta_{0}}{2} x_{*}^{2} .
\end{aligned}
$$

\section{2D case}

In the axisymmetric system (2D), we follow a similar procedure. However, extra terms connected to the different expression of the curvature appear. Following the same steps as for the derivation of Eq. (D1), we Eq. (69b) two times. Then, given the parity condition and the expansion of $\tilde{U}$ for small $\zeta$, we obtain

$$
\begin{aligned}
0= & \frac{r^{2}}{4 D_{e}} u_{C z}-\frac{A}{2 \lambda}\left(\zeta^{2}-\zeta_{0}^{2}\right)+\gamma\left[\zeta \partial_{r r} \zeta-2 \zeta_{0} \partial_{r r} \zeta_{0}\right. \\
& \left.-\frac{1}{2}\left(\partial_{r} \zeta\right)^{2}+\frac{\zeta \partial_{r} \zeta}{r}-\int_{0}^{r} d r^{\prime} \frac{\left(\partial_{r^{\prime}} \zeta\right)^{2}}{r^{\prime}}\right] .
\end{aligned}
$$

To derive an explicit expression for the correction to $\zeta_{\text {sing }}$, we insert its expression Eq. (62b) plus a perturbation $\delta \zeta$ in Eq. (D4). Thus, Eq. (D4) becomes

$$
\begin{aligned}
0= & -\frac{A}{\lambda^{2}} \omega r \delta \zeta-\gamma\left[\left(\partial_{r r} \zeta_{0}\right)^{2} \frac{r_{*}^{2}}{2}+2 \zeta_{0} \partial_{r r} \zeta_{0}\right] \\
& +\gamma\left[\frac{1}{2} \omega^{2}-\omega^{2} \ln \left(\frac{r}{r_{*}}\right)+\omega \frac{\delta \zeta}{r}\right. \\
& \left.+2 \omega \int_{r_{*}}^{r} d r \frac{\partial_{r} \delta \zeta}{r}+\omega r \partial_{r r} \delta \zeta\right],
\end{aligned}
$$

where now in $2 \mathrm{D}$

$$
\omega=\left(\frac{u_{C z}}{2 D_{e} A}\right)^{\frac{1}{2}} \lambda .
$$

Note that the integral term of Eq. (D4) was rewritten as follows:

$$
\begin{aligned}
\int_{0}^{r} d r \frac{\left(\partial_{r} \zeta\right)^{2}}{r}= & \int_{0}^{r_{*}} d r \frac{\left(\partial_{r} \zeta_{\mathrm{tip}}\right)^{2}}{r}+\int_{r_{*}}^{r} d r \frac{\left(\partial_{r} \zeta_{\text {sing }}\right)^{2}}{r} \\
= & -\gamma\left(\partial_{r r} \zeta_{0}\right)^{2} \frac{r_{*}^{2}}{2}+\omega^{2} \ln \left(\frac{r}{r_{*}}\right) \\
& +2 v \int_{r_{*}}^{r} d r \frac{\partial_{r} \delta \zeta}{r} .
\end{aligned}
$$

If in the matching procedure, for $r \gg r_{*}$, we keep only dominant terms, as done above for the 1D case, we would obtain $\delta \zeta \approx 1 / r$. Since this does not behave properly we make a crude approximation and keep only one higher order term $\omega \delta \zeta / r$ to account for higher order contributions in Eq. (D5) (indeed, as $r \rightarrow r_{*}$ the integral term vanishes and we expect the other relevant term $r \partial_{r r} \delta \zeta$ to be of the same order as $\omega \delta \zeta / r$ ). With these assumptions we find

$$
\delta \zeta=\gamma \frac{\frac{1}{2} \omega^{2}-2 \zeta_{0} \partial_{r r} \zeta_{0}-\omega^{2} \ln \frac{r}{r_{*}}-\frac{1}{2}\left(\partial_{r r} \zeta_{0}\right)^{2} r_{*}^{2}}{\frac{A}{\lambda^{2}} \omega r-\gamma \omega \frac{1}{r}} .
$$

As before, to obtain the matching between the two solutions $\zeta_{\text {tip }}$ and $\zeta_{\text {sing }}$ we use two conditions. First, we consider the matching of the thicknesses $\omega r_{*}+\delta \zeta\left(r_{*}\right)=\zeta_{0}+\partial_{r r} \zeta_{0} r_{*}^{2} / 2$. A second relation accounts for the matching of the slopes $\omega+\left.\partial_{r} \delta \zeta(r)\right|_{r_{*}}=\partial_{r r} \zeta_{0} r_{*}$. Since in this case $\partial_{r} \delta \zeta(r)$ does not diverge for $r \rightarrow 0$, and since $r_{*}$ is assumed to be small, we

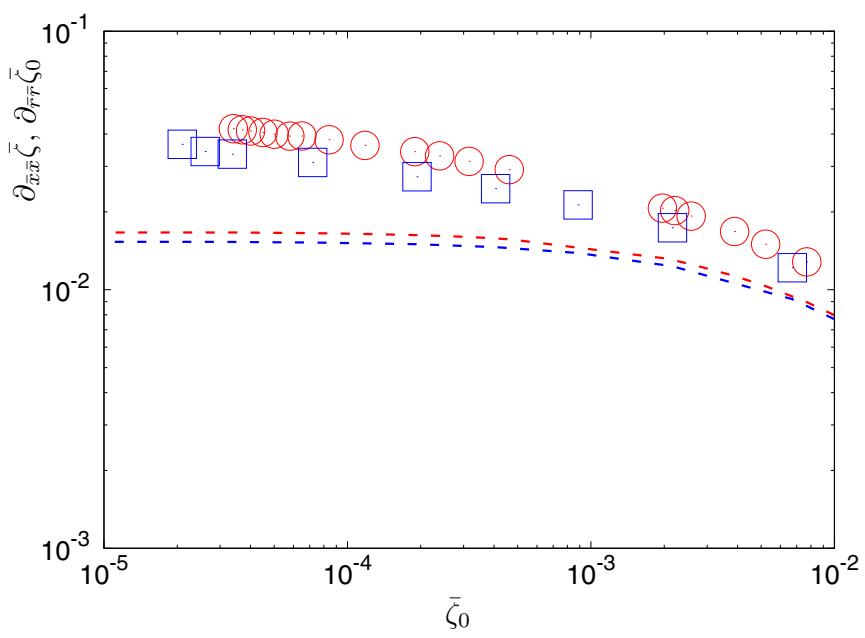

FIG. 11. Curvature at the tip as a function of the tip-substrate gap $\bar{\zeta}_{0}$. Red circles: simulations result for the wedge contact (1D); blue squares: simulations result for the axisymmetric conical contact (2D). Sizes of the simulation boxes are $\bar{L}=\bar{R}=100$; dashed red and blue lines analytical prediction using the solution of Eq. (D7) and Eq. (D3), respectively, using the the assumption $L_{c} \approx L, R_{c} \approx R$. The results are in normalized units.

neglect the contribution $\left.\partial_{r} \zeta\right|_{r_{*}}$ in the slope. This lead to the following system of equations:

$$
\begin{gathered}
\omega r_{*}+\gamma\left[\frac{\omega^{2}}{2}-2 \zeta_{0} \partial_{r r} \zeta_{0}-\frac{\left(\partial_{r r} \zeta_{0}\right)^{2}}{2} r_{*}^{2}\right]\left(\frac{A}{\lambda^{2}} \omega r_{*}-\gamma \eta \frac{1}{r_{*}}\right)^{-1} \\
=\zeta_{0}+\partial_{r r} \zeta_{0} \frac{r_{*}^{2}}{2}, \\
\partial_{r r} \zeta_{0} r_{*}=\omega .
\end{gathered}
$$

\section{Numerical solution}

Inserting the asymptotic analytical expression of the dissolution rate $u_{C z}$, Eq. (61), we solved the linear systems of Eqs. (D3) and (D7) using MINPACK routine [60]. We obtain values of $x_{*}\left(r_{*}\right)$ and of $\partial_{x x} \zeta_{0}\left(\partial_{r r} \zeta_{0}\right)$ for a given minimum distance $\zeta_{0}$. The results, displayed in Fig. 11, are represented by the dashed lines and compared with the simulation results. In particular, we find (in normalized units) for $\zeta_{0}=0, \partial_{\bar{x} \bar{x}} \bar{\zeta}_{0} \approx$ 0.0167 and $\partial_{\bar{r} \bar{r}} \bar{\zeta}_{0} \approx 0.0153$.

\section{APPENDIX E: BEYOND THE LINEARIZATION OF THE GIBBS-THOMSON RELATION}

A simple substitution allows one to include the effect of the exponential term in the analysis of the contact profile in the absence of surface tension:

$$
U^{\prime}(\zeta) \rightarrow k_{B} T \exp \left[\frac{U^{\prime}(\zeta)}{k_{B} T}\right] .
$$

This leads to a different definition of $\tilde{U}$ from the relation

$$
\tilde{U}^{\prime}(\zeta)=\zeta U^{\prime \prime}(\zeta) \exp \left[\frac{U^{\prime}(\zeta)}{k_{B} T}\right] .
$$

The same procedure as that discussed in Sec. IV can then be applied with this new expression for $\tilde{U}$. 
For power-law potentials, this leads to an essential singularity in $\tilde{U}$ when $\zeta \rightarrow 0$ :

$$
\tilde{U}(\zeta)=\zeta k_{B} T\left[e^{-\frac{A n \zeta^{-n-1}}{k_{B} T}}-\frac{E_{1+\frac{1}{n+1}}\left(\frac{A n \zeta^{-n-1}}{k_{B} T}\right)}{n+1}\right],
$$

where

$$
E_{m}(z)=\int_{1}^{\infty} d t \frac{\mathrm{e}^{-z t}}{t^{m}}
$$

This essential singularity appears in the relation between $u_{C z}$ and the minimum thickness $\zeta_{0}$ when $\zeta_{0} \rightarrow 0$ :

$$
u_{C z}=4 D_{e}\left[\tilde{U}\left(\zeta_{0}\right)-\tilde{U}(\infty)\right] .
$$

In contrast, there is no significant change in the case of an exponential potential. Indeed, the central property of being finite when $\zeta_{0} \rightarrow 0$ is not affected by Eq. (E1). Thus, the exponential potential again leads to a pointy shape, and constant dissolution rate obeys Eq. (61). Moreover, the details of the regularization of the tip due to surface tension can be affected but we do not expect major changes.
[1] J. Lavalle, Compte Rend. Acad. Sci. (Paris) 36, 493 (1853).

[2] H. C. Sorby, Philos. Mag. Ser. 4 11, 20 (1856).

[3] G. F. Becker and A. L. Day, J. Geol. 24, 313 (1916).

[4] S. Taber, Am. J. Sci. 41, 532 (1916).

[5] C. W. Correns and W. Steinborn, Zeitschr. Kristallogr.-Cryst. Mat. 101, 117 (1939).

[6] G. J. Ramsay, Folding and Fracturing of Rocks (McGraw-Hill, New York, 1967), p. 568.

[7] D. W. Durney, Nature (London) 235, 315 (1972).

[8] J. Desarnaud, D. Bonn, and N. Shahidzadeh, Sci. Rep. 6, 30856 (2016).

[9] G. W. Scherer, Cem. Concr. Res. 29, 1347 (1999).

[10] R. J. Flatt, J. Cryst. Growth 242, 435 (2002).

[11] M. Steiger, J. Cryst. Growth 282, 470 (2005).

[12] P. K. Weyl, J. Geophys. Res. 64, 2001 (1959).

[13] R. L. Coble, J. Appl. Phys. 34, 1679 (1963).

[14] D. Elliott, Geol. Soc. Am. Bull. 84, 2645 (1973).

[15] E. H. Rutter, Philos. Trans. R. Soc. A 283, 203 (1976).

[16] R. Raj, J. Geophys. Res. 87, 4731 (1982).

[17] V. Kruzhanov and B. Stöckhert, Pure Appl. Geophys. 152, 667 (1998).

[18] A. C. Fowler and X. Yang, J. Geophys. Res. 104, 12989 (1999).

[19] J. N. Israelachvili, Intermolecular and Surface Forces, 3rd ed. (Academic Press, New York, 1991), p. 710.

[20] E. J. W. Verwey, J. Phys. Chem. 51, 631 (1947).

[21] N. Alcantar, J. Israelachvili, and J. Boles, Geochim. Cosmochim. Acta 67, 1289 (2003).

[22] S. Veeramasuneni, Y. H. Hu, M. R. Yalamanchili, and J. D. Miller, J. Colloid Interface Sci. 188, 473 (1997).

[23] C. Drummond and J. Israelachvili, J. Petroleum Sci. Eng. 33, 123 (2002).

[24] M. Dishon, O. Zohar, and U. Sivan, Langmuir 25, 2831 (2009).

[25] Y. Diao and R. M. Espinosa-Marzal, Proc. Natl. Acad. Sci. U.S.A. 113, 12047 (2016).

[26] J. J. Valle-Delgado, J. A. Molina-Bolívar, F. Galisteo-González, M. J. Gálvez-Ruiz, A. Feiler, and M. W. Rutland, J. Chem. Phys. 123, 034708 (2005).

[27] A. Hamilton, V. Koutsos, and C. Hall, Chem. Commun. 46, 5235 (2010).

[28] R. Tönhardt and G. Amberg, J. Cryst. Growth 194, 406 (1998).

[29] W. R. Wilcox, J. Cryst. Growth 65, 133 (1983).
[30] B. J. Hamrock, NASA Report 1255, Tech. Rep. (1991).

[31] D. Medvedev and K. Kassner, Phys. Rev. E 72, 056703 (2005).

[32] Ø. Hammer, D. K. Dysthe, B. Lelu, H. Lund, P. Meakin, and B. Jamtveit, Geochim. Cosmochim. Acta 72, 5009 (2008)

[33] Y. Tao, A. Yeckel, and J. J. Derby, J. Comp. Phys. 315, 238 (2016).

[34] A. Oron and S. G. Bankoff, Rev. Mod. Phys. 69, 931 (1997).

[35] T. E. Karis, W. T. Kim, and M. S. Jhon, Tribol. Lett. 18, 27 (2005).

[36] O. K. Matar, V. Gkanis, and S. Kumar, J. Colloid Interface Sci. 286, 319 (2005).

[37] M. J. Blount, M. J. Miksis, and S. H. Davis, Proc. IUTAM 16, 33 (2015).

[38] T. Le Goff, P. Politi, and O. Pierre-Louis, Phys. Rev. E 90, 032114 (2014).

[39] C. D. M. Etienne Guyon, J.-P. Hulin, and L. Petit, Physical Hydrodynamics, 2nd ed. (Oxford University Press, Oxford, UK, 2015).

[40] Y. Saito, Statitical Physics of Crystal Growth (World Scientific, Singapore, 1996).

[41] D. Quere, F. Brochard-Wyart, and P. G. de Gennes, Capillarity and Wetting Phenomena (Springer, Berlin, 2003).

[42] C. Weatherburn, Differential Geometry of Three Dimensions, 4th ed. (Cambridge University Press, Cambridge, UK, 1955).

[43] R. Goldman, Comp. Aided Geometric Design 22, 632 (2005).

[44] L. N. Plummer and T. M. L. Wigley, Geochim. Cosmochim. Acta 40, 191 (1976).

[45] L. Yuan-Hui and S. Gregory, Geochim. Cosmochim. Acta 38, 703 (1974).

[46] E. V. D. Voort and P. Hartman, J. Cryst. Growth 89, 603 (1988).

[47] W. Wang and W. Hu, J. Cryst. Growth 160, 398 (1996).

[48] C. Misbah, F. Renard, J.-P. Gratier, and K. Kassner, Geophys. Res. Lett. 31, L06618 (2004).

[49] J. Wonham, Nature (London) 215, 1053 (1967).

[50] M. Wang, C.-C. Chang, and R.-J. Yang, J. Chem. Phys. 132, 024701 (2010).

[51] J. Høgberget, A. Røyne, D. K. Dysthe, and E. Jettestuen, Phys. Rev. E 94, 023005 (2016).

[52] E. A. Pachon-Rodriguez, A. Piednoir, and J. Colombani, Phys. Rev. Lett. 107, 146102 (2011). 
[53] A. Pimpinelli and J. Villain, Physics of Crystal Growth (Cambridge University Press, Cambridge, UK, 1998).

[54] X. Zhang and C. Spiers, Int. J. Rock Mechanics Mining Sci. 42, 950 (2005).

[55] J.-P. Gratier, D. Dysthe, and F. Renard, Adv. Geophys. 54, 47 (2013).

[56] B. Den Brok, M. Zahid, and C. W. Passchier, Tectonophysics 307, 297 (1999).
[57] D. Croizé, F. Renard, K. Bjørlykke, and D. K. Dysthe, J. Geophys. Res.: Solid Earth 115, B09207 (2010).

[58] S. F. Cox and M. S. Paterson, Geophys. Res. Lett. 18, 1401 (1991).

[59] D. Blackmore and L. Ting, SIAM Rev. 27, 569 (1985).

[60] J. J. Moré, B. S. Garbow, and K. E. Hillstrom, User Guide for MINPACK-1, Tech. Rep. ANL-80-74 (Argonne National Laboratory, 1980). 